\title{
A DIFFUSION MODEL FOR TWO PARALLEL QUEUES WITH PROCESSOR SHARING: TRANSIENT BEHAVIOR AND ASYMPTOTICS
}

\author{
CHARLES KNESSL \\ University of Illinois at Chicago \\ Dept. of Mathematics, Statistics, and Computer Science (M/C 249) \\ Chicago, IL 60607 USA \\ e-mail:knessl@uic.edu
}

(Received April, 1998; Revised February, 1999)

\begin{abstract}
We consider two identical, parallel $M / M / 1$ queues. Both queues are fed by a Poisson arrival stream of rate $\lambda$ and have service rates equal to $\mu$. When both queues are non-empty, the two systems behave independently of each other. However, when one of the queues becomes empty, the corresponding server helps in the other queue. This is called head-of-the-line processor sharing. We study this model in the heavy traffic limit, where $\rho=\lambda / \mu \rightarrow 1$. We formulate the heavy traffic diffusion approximation and explicitly compute the time-dependent probability of the diffusion approximation to the joint queue length process. We then evaluate the solution asymptotically for large values of space and/or time. This leads to simple expressions that show how the process achieves its stead state and other transient aspects.
\end{abstract}

Key words: Processor-Shared Queues, Diffusion Model, Transient Distribution.

AMS subject classifications: $60 \mathrm{~K} 25,90 \mathrm{~B} 22,60 \mathrm{~J} 60$.

\section{Introduction}

Queueing systems are used in a wide variety of applications, such as computer and communications networks and manufacturing systems. In analyzing such models, one typically wishes to compute the probability distribution of some stochastic process. Obtaining the full time-dependent distribution is a difficult task for all but simple models.

Here we consider the following model, which is sometimes referred to as head-ofthe-line processor sharing of parallel queues. There are two parallel $M / M / 1$ queues, each fed by independent Poisson arrival streams with rate $\lambda$. Each of the two servers works at rate $\mu$. When both queues are non-empty, each server tends to its own queue. However, if the first (resp., second) queue becomes empty, the first (resp., 
second) server helps the server in the other queue, thereby providing a service rate of $2 \mu$ during the idle period. The steady state joint queue length distribution for this model was analyzed by Konheim, Meilijson and Melkman [11] and a more general model which allows, e.g., for different service rates was analyzed by Fayolle and Iasnogorodski [6]. In [11], the authors obtained an expression for the two-dimensional generating function of the joint queue-length distribution in terms of elliptic integrals. The inherent complexity of the solutions in [11] and [6] led other authors to investigate asymptotic properties of these solutions, in order to gain more qualitative insights.

The heavy traffic limit is defined as $\rho=\lambda / \mu \rightarrow 1$. In this limit, the joint queue length process $\left(N_{1}(t), N_{2}(t)\right)\left(N_{j}(t)=\right.$ number of customers in the $j$ th queue) may be approximated by a diffusion process, whose analysis proves to be simpler than that of the discrete model. In particular, Knessl [9] and Morrison [12] obtained a relatively simple answer for the steady state distribution of the diffusion approximation. Also, some exact [12] and asymptotic [9] results were obtained for more general models, which allow different service rates and discriminatory processor-sharing. Diffusion approximations were also used by Fendick and Rodrigues [7] to treat more complicated models, with more than two queues. Related models with finite capacity were analyzed by Morrison [13]. Applications of these models include the buffering of channels (i.e. virtual circuits) in wide-area data networks (see [13]).

In this paper we compute the time-dependent distribution for the heavy traffic diffusion model, thereby obtaining information on how steady state is achieved, and other transient phenomena. Denoting by $p(m, n, t)$ the joint probability that $N_{1}(t)=m$ and $N_{2}(t)=n$, we obtain (in the stable case) the approximation $p(m, n, t) \sim \epsilon^{2} P(x, y, T)$, where $\epsilon=1-\rho$ and $(x, y, T)=\left(\epsilon m, \epsilon n, \mu \epsilon^{2} t\right)$ are scaled space and time variables. We shall obtain explicit, albeit complicated, expressions for $P$ and then evaluate these asymptotically for various ranges of space and time. This leads to very simple formulas that clearly show the basic qualitative structure of the joint density function.

In particular, we show that there is a surface $T=T_{*}(x, y)$ in the $(x, y, T)$ space so that for $T>T_{*}$, the process has settled to its steady state distribution, while for $T<T_{*}$, the probability distribution depends upon time as well as on the initial conditions. We shall also consider the unstable case where $\rho>1$ in the heavy traffic limit. Here the process is transient and the queue lengths tend to grow without bound. We shall show (cf. Theorem 3(a) and (3.10)) that the two queues are nevertheless coupled in this limit, and obtain a simple quantitative measure of this interaction.

In the asymptotic analysis, we shall allow for space $(x, y)$ to be large as well as time $T$. We contrast this to "relaxation rate" asymptotics, which are discussed in the book of Cohen [5] for single server queues and by Blanc [2] for two tandem $M / M / 1$ queues. These asymptotics (for the diffusion model) would assume that the space variables are held fixed and use the approach to equilibrium in the form $P(x, y, T)-P_{e q}(x, y) \sim T^{\nu} e^{-\alpha T} \beta(x, y)$, where $\alpha$ ( = relaxation rate) and $\nu$ are constants. Here $P_{e q}$ is the equilibrium density, which is non-zero only if $\rho<1$. We believe that the asymptotics presented here give a more global description of the transient distribution. We have previously obtained analogous asymptotic results for various models with one space dimension (see Knessl [10], Xie and Knessl [23], and Tan and Knessl [18]). In the probability literature, these types of asymptotics are sometimes referred to as "large deviations theory." However, such theory generally only gives the exponential rate of growth or decay of the desired quantity. In contrast, 
here we give very precise results and also indicate how to obtain full asymptotic series. We obtain the results by the saddle point and related methods for evaluating integrals [3].

We also mention related work on the $M / M / 1$-PS queue and more general models by Coffman et al. [4], Ramaswami [16], Yashkov [20-22], Ott [15], and Sengupta [17]. These authors consider single queues with the processor sharing service discipline. The main focus in these papers is the calculation of the sojourn time distribution of a tagged customer, as well as its moments. The queue length distribution in the $M / M / 1$-PS model is the same for the PS and FIFO service disciplines. It is the sojourn time distribution that is very different for PS and FIFO service. If we condition the sojourn time on the total service that the tagged customer must receive, then the PS discipline is much more efficient at servicing shorter jobs. In [4], the authors analyzed the $M / M / 1$-PS model and computed the Laplace transform of the sojourn time distribution, conditioned on the job size. In [16], the $G I / M / 1$-PS model is analyzed. In particular, simple expressions are given for the first two (unconditional) sojourn time moments. The $M / G / 1$-PS model was analyzed by Yashkov [20-22] and Ott [15]. The response time distribution is computed in [20], the transient distribution of the number of customers present is analyzed in [21] and a good survey of work on processor-shared queues appears in [22]. In [15], the author extended the results in [20] to calculate the joint distribution of the sojourn time and of the number of customers present upon the departure of the tagged customer. Some approximations for the more difficult $G I / G / 1-\mathrm{PS}$ model are given in [17].

We believe that the structure of $P(x, y, T)$ revealed here (both exact and asymptotic) will also arise in other diffusion (and also discrete) models corresponding to two or more coupled queues. Other explicit solutions to diffusion models arising in queueing networks are given in Newell [14], Foschini [8], Knessl and Tan [19] and Avram [1]. In [1], the author classifies the steady-state densities of a large class of two-dimensional diffusion models according to their tail behaviors as $x$ and/or $y \rightarrow \infty$. We believe that such a classification should also be possible for the transient behavior, and this work may be viewed as a first step in that direction. We also mention that the diffusion approximation analysis presented here should be extendible to problems with general arrivals and/or service. However, these generalizations are likely to lead to somewhat more complicated PDEs and boundary conditions (BCs) then those in (2.10)-(2.12).

In Section 2, we formulate the model. In Section 3, we summarize and briefly discuss our main results. The detailed calculations are presented in Sections 4 and 5.

\section{Statement of the Problem}

We let $N_{1}(t)$ (resp., $N_{2}(t)$ ) be the number of customers in the first (resp., second) queue. We define the transition probability distribution by

$$
\begin{aligned}
p(m, n, t) \quad & =p\left(m, n, t ; m_{0}, n_{0}\right) \\
& =\operatorname{Prob}\left[N_{1}(t)=m, N_{2}(t)=n \mid N_{1}(0)=m_{0}, N_{2}(0)=n_{0}\right] .
\end{aligned}
$$

In terms of $(m, n)$, the distribution $(2.1)$ satisfies the forward equation (see also [9, 11, 12]) 


$$
\begin{aligned}
p_{t}(m, n, t)= & \lambda[p(m-1, n, t)+p(m, n-1, t)-2 p(m, n, t)] \\
& +\mu[p(m+1, n, t)+p(m, n+1, t)-2 p(m, n, t)]
\end{aligned}
$$

with the boundary conditions

$$
\begin{aligned}
p_{t}(m, 0, t)= & \lambda[p(m-1,0, t)-2 p(m, 0, t)] \\
& +2 \mu[p(m+1,0, t)-p(m, 0, t)]+\mu p(m, 1, t) \\
p_{t}(0, n, t) \quad & \lambda[p(0, n-1, t)-2 p(0, n, t)] \\
& +2 \mu[p(0, n+1, t)-p(0, n, t)]+\mu p(1, n, t),
\end{aligned}
$$

the corner condition

$$
p_{t}(0,0, t)=2 \mu[p(1,0, t)+p(0,1, t)]-2 \lambda p(0,0, t)
$$

and the initial condition

$$
p(m, n, 0)=\delta\left(m, m_{0}\right) \delta\left(n, n_{0}\right)
$$

Here subscripts denote partial derivatives and $\delta$ is the Kronecker delta symbol.

We study the model in the heavy traffic limit, where $\rho=\lambda / \mu \rightarrow 1$. Formally, we define the small positive parameter $\epsilon$ by

$$
1-\rho=1-\frac{\lambda}{\mu}=\epsilon a
$$

and scale space and time by $\epsilon$ as follows

$$
m=\frac{x}{\epsilon}, n=\frac{y}{\epsilon}, t=\frac{T}{\mu \epsilon^{2}}, m_{0}=\frac{x_{0}}{\epsilon}, n_{0}=\frac{y_{0}}{\epsilon} .
$$

Note that this means that the initial queue lengths are assumed to be large, of the order $O\left(\epsilon^{-1}\right)$.

If the queue is stable (i.e., $\rho<1$ ), we will set $a=+1$, and then (2.7) defines $\epsilon$ in terms of $\rho$. In the unstable case $(\rho>1)$, we will set $a=-1$. If $\rho=1$, we take $a=0$ and then $(2.8)$ corresponds to viewing $p(m, n, t)$ on large space/time scales, with $m, n, t \rightarrow \infty$ and $m / \sqrt{t}, n / \sqrt{t}$ fixed.

With (2.8), we expand the probability distribution as

$$
p(m, n, t)=\epsilon^{2}\left[P(x, y, T)+\epsilon P^{(1)}(x, y, T)+\ldots\right] .
$$

From (2.2)-(2.4) and (2.6), we find that the leading term $P$ satisfies the PDE (see also $[9,12])$

$$
P_{T}=P_{x x}+P_{y y}+a\left(P_{x}+P_{y}\right) ; x, y, T>0
$$

the boundary conditions (BC) 


$$
\begin{aligned}
& P_{x}(0, y, T)+P_{y}(0, y, T)+a P(0, y, T)=0 ; y, T>0 \\
& P_{x}(x, 0, T)+P_{y}(x, 0, T)+a P(x, 0, T)=0 ; x, T>0
\end{aligned}
$$

and the initial condition

$$
P(x, y, 0)=\delta\left(x-x_{0}\right) \delta\left(y-y_{0}\right)
$$

We also have the normalization condition

$$
\int_{0}^{\infty} \int_{0}^{\infty} P(x, y, T) d x d y=1 \text { for all } T \geq 0
$$

We do not consider the corner condition (2.5) in formulating the heavy traffic diffusion model. We will show that $P(x, y, T)$ becomes infinite near the origin $x=$ $y=0$, and hence (2.9) cannot be the correct asymptotic approximation to the discrete probabilities $p(m, n, t)$ for small values of $x$ and $y$ (more precisely for $(x, y)=O(\epsilon)$, which is the same as $(m, n)=O(1))$. A proper analysis of the corner region would involve analyzing the discrete model (2.2)-(2.5), with $\mu=\lambda+\mu \epsilon a$. However, we will show that such a detailed treatment is not necessary to determine $P(x, y, T)$, which is the heavy traffic diffusion approximation valid away from the corner. The total probability mass in the corner region is asymptotically smaller than that on the $(x, y)$ scale. However, calculating the higher order terms in the series (2.9) (e.g., the function $\left.P^{(1)}\right)$ would necessitate a careful treatment of the corner region.

We shall obtain an explicit solution for the leading order diffusion approximation $P(x, y, T)$. Then we shall obtain detailed asymptotic results for this limiting density, that apply for $x$ and/or $y$ and/or $T$ large. The final results are summarized in Section 3 and the details of the calculations are given in Sections 4 and 5 .

\section{Summary of Main Results}

In Section 4 we solve (2.10)-(2.14) and obtain the following integral representations for $P(x, y, T)$.

Theorem 1: The transient solution to the diffusion model is

$$
P(x, y, T)=P_{I}(x, y, T)+P_{I I}(x, y, T)
$$

where

$$
\begin{gathered}
P_{I}=\frac{1}{4 \pi T} \exp \left[-\frac{a^{2}}{2} T-\frac{a}{2}\left(x-x_{0}+y-y_{0}\right)\right] \\
\times\left\{\exp \left[-\frac{\left(x-x_{0}\right)^{2}+\left(y-y_{0}\right)^{2}}{4 T}\right]-\exp \left[-\frac{\left(x+x_{0}\right)^{2}+\left(y+y_{0}\right)^{2}}{4 T}\right]\right. \\
+\frac{x+x_{0}-y+y_{0}}{2 T} \int_{0}^{\infty} \exp \left[-\frac{\left(x+u+x_{0}\right)^{2}+\left(y+u-y_{0}\right)^{2}}{4 T}\right] d u
\end{gathered}
$$




$$
\left.+\frac{-x+x_{0}+y+y_{0}}{2 T} \int_{0}^{\infty} \exp \left[-\frac{\left(x+u-x_{0}\right)^{2}+\left(y+u+y_{0}\right)^{2}}{4 T}\right] d u\right\}
$$

and

$$
\begin{gathered}
P_{I I}=\frac{\sqrt{2}}{\pi} \exp \left[\frac{a}{2}\left(x_{0}+y_{0}-x-y\right)\right] \int_{0}^{T} \int_{0}^{\infty} \int_{0}^{\infty} \exp \left[-\frac{a^{2}}{2} \tau-\frac{k^{2}}{4 \tau}\right] \\
\times \frac{(x+u)(y+u)}{\zeta^{3}}\left[\frac{1}{16 \sqrt{\pi}} \frac{1}{\tau^{5 / 2}} H_{4}\left(\frac{k}{2 \sqrt{\tau}}\right)+\frac{a}{8 \sqrt{2 \pi}} \frac{1}{\tau^{2}} H_{3}\left(\frac{k}{2 \sqrt{\tau}}\right)\right] d \zeta d u d \tau \\
k=\frac{x_{0}+y_{0}}{\sqrt{2}}+\frac{\zeta}{2}+\frac{(x+u)^{2}+(y+u)^{2}}{2 \zeta} .
\end{gathered}
$$

Here $H_{j}(\cdot)$ are Hermite polynomials, hence

$$
H_{3}(z)=8 z^{3}-12 z, \quad H_{4}(z)=16 z^{4}-48 z^{2}+12 .
$$

Alternate expressions for $P_{I I}$ are

$$
P_{I I}=\frac{1}{2 \pi i} \int_{\mathrm{Br}} e^{\theta T} \widehat{P}_{I I}(x, y ; \theta) d \theta
$$

where

$$
\begin{aligned}
& \widehat{P}_{I I}=\frac{2 \theta+a^{2}}{\pi \theta}\left[\sqrt{a^{2}+2 \theta}+a\right] e^{-a(x+y) / 2} \exp \left[\left(x_{0}+y_{0}\right)\left(\frac{a}{2}-\sqrt{\frac{\theta}{2}+\frac{a^{2}}{4}}\right)\right] \\
& \times \int_{0}^{\infty} \frac{(x+u)(y+u)}{(x+u)^{2}+(y+u)^{2}} K_{2}\left(\sqrt{\theta+\frac{a^{2}}{2}} \sqrt{(x+u)^{2}+(y+u)^{2}}\right) d u
\end{aligned}
$$

or

$$
\begin{aligned}
\widehat{P}_{I I} & =\frac{\sqrt{a^{2}+2 \theta}+a}{2 \pi \theta} \sqrt{\theta+\frac{a^{2}}{2}} e^{-a(x+y) / 2} \exp \left[\left(x_{0}+y_{0}\right)\left(\frac{a}{2}-\sqrt{\frac{\theta}{2}+\frac{a^{2}}{4}}\right)\right] \\
& \times \int_{-\infty}^{\infty} \frac{i \sinh (2 \eta)}{\cosh \eta+i \sinh \eta} \exp \left[-\sqrt{\theta+\frac{a^{2}}{2}}(x \cosh \eta+i y \sinh \eta)\right] d \eta .
\end{aligned}
$$

Here $K_{2}(\cdot)$ is the modified Bessel function of order 2 and $B r$ is a vertical contour in the complex $\theta$-plane, on which $\operatorname{Re}(\theta)>0$.

We note that $\widehat{P}_{I I}$ is the Laplace transform of $P_{I I}$. When $a>0$ (i.e., $\rho<1$ ), $\widehat{P}_{I I}$ has simple pole at $\theta=0$, which determines the limiting behavior of $P_{I I}$ as $T \rightarrow \infty$ :

$$
\begin{gathered}
P_{I I}(x, y, \infty)=\lim _{\theta \rightarrow 0}\left[\theta \widehat{P}_{I I}(x, y ; \theta)\right] \\
=\frac{2 a^{3}}{\pi} e^{-a(x+y) / 2} \int_{0}^{\infty} \frac{(x+u)(y+u)}{(x+u)^{2}+(y+u)^{2}} K_{2}\left(\frac{a}{\sqrt{2}} \sqrt{(x+u)^{2}+(y+u)^{2}}\right) d u .
\end{gathered}
$$

As $T \rightarrow \infty$, we have $P_{I} \rightarrow 0$ and thus (3.7) gives the steady-state density of the 
diffusion approximation, and this agrees with the result previously obtained by Knessl [9] and Morrison [12]. When $a \leq 0$ (i.e., $\rho \geq 1$ ), the poles is absent and we now have $P_{I}, P_{I I} \rightarrow \infty$ as $T \rightarrow \infty$.

Now observe that the total number of customers $N_{1}(t)+N_{2}(t)$ in the two queue network benaves as the standard $M / M / 1$ model with an arrival rate $=2 \lambda$ and service rate $=2 \mu$. In appendix $\mathrm{A}$, we show after a lengthy calculation that

$$
\begin{gathered}
\int_{0}^{z} \widehat{P}(w, z-w ; \theta) d w=\frac{e^{-a\left(z-z_{0}\right) / 2}}{2 \sqrt{2 \theta+a^{2}}}\left\{\exp \left[-\frac{\left|z-z_{0}\right|}{\sqrt{2}} \sqrt{\theta+\frac{a^{2}}{2}}\right]\right. \\
\left.+\frac{\sqrt{2 \theta+a^{2}}+a}{\sqrt{2 \theta+a^{2}}-a} \exp \left[-\frac{z+z_{0}}{\sqrt{2}} \sqrt{\theta+\frac{a^{2}}{2}}\right]\right\}
\end{gathered}
$$

where $z_{0}=x_{0}+y_{0}$ and $\widehat{P}$ is the Laplace transform of $P(x, y, T)$ over time.

We denote by $(X(T), Y(T))$ the diffusion process that approximates our discrete queue. Then the density of $X(T)+Y(T)$ should be precisely that of the heavy traffic diffusion approximation to the standard $M / M / 1$ model. Denoting this density by $\mathscr{P}(z, T)$, it satisfies

$$
\begin{gathered}
{ }_{2}^{\frac{1}{2} \mathscr{P}_{T}}=\mathscr{P}_{z z}+a \mathscr{P}_{z} ; z, T>0 \\
\mathscr{P}_{z}+a \mathfrak{P}=0 ; z=0, T>0 \\
\left.\mathscr{P}\right|_{T=0}=\delta\left(z-z_{0}\right) .
\end{gathered}
$$

The factor of $\frac{1}{2}$ in the time-derivative of (3.9) arises due to the fact that the discrete model has a total arrival rate $=2 \lambda$, rather than $\lambda$. We let $\widehat{\mathscr{P}}(z ; \theta)=$ $\int_{0}^{\infty} e^{-\theta T \mathscr{P}}(z, T) d T$. Then, solving (3.9) for the Laplace transform $\hat{z}$, we obtain precisely the right side of $(3.8)$. This shows that $\widehat{\mathscr{P}}(z ; \theta)=\int_{0}^{z} \widehat{P}(w, z-w ; \theta) d w$, and
this must be the case.

Since the solution in Theorem 1 is quite complicated, we evaluate it asymptotically for large values of space and/or time. This yields simpler formulas that show more clearly the basic qualitative features of the joint density. First we take initial conditions $x_{0}=y_{0}=0$. In view of $(2.8)$, this does not mean that the discrete process $\left(N_{1}(t), N_{2}(t)\right)$ starts at the origin $(0,0)$, but rather that $m_{0}$ and $n_{0}$ are of order $o\left(\epsilon^{-1}\right)$. When $x_{0}=y_{0}=0$, we have $P_{I}=0$ and hence $P=P_{I I}$. The following results are established in Section 5.

Theorem 2: For $x_{0}=y_{0}=0$ and $\rho<1$, we set $a=+1$. Asymptotic expansions

of $P(x, y, T)$ are as follows:
\[ (a) \quad x, y, T \rightarrow \infty \text { with } T<T_{*}(x, y) \equiv \sqrt{\frac{x^{2}+y^{2}}{2}} \]

$$
\begin{gathered}
P(x, y, T) \sim K(x, y, T) \exp [\Psi(x, y, T)] \\
\Psi(x, y, T)=-\frac{x+y+T}{2}-\frac{x^{2}+y^{2}}{4 T}
\end{gathered}
$$




$$
K(x, y, T)=\frac{\sqrt{2}}{\pi} \frac{x y}{T(x+y)} \frac{1}{\sqrt{x^{2}+y^{2}}-\sqrt{2} T}
$$

(b) $\quad x, y, T \rightarrow \infty$ with $T>T_{*}(x, y)$

$$
P(x, y, T) \sim \frac{1}{\sqrt{\pi}} \frac{2^{5 / 4} x y}{(x+y)\left(x^{2}+y^{2}\right)^{3 / 4}} \exp \left[-\frac{1}{2}(x+y)-\frac{1}{\sqrt{2}} \sqrt{x^{2}+y^{2}}\right]
$$

(c) $\quad x, y, T \rightarrow \infty$ with $T \approx T_{*}(x, y)\left(\right.$ more precisely $\left.T-T_{*}=O(\sqrt{x})\right)$

$$
P(x, y, T)
$$

$$
\begin{gathered}
\sim \frac{2^{5 / 4}}{\sqrt{\pi}} \frac{x y}{\left(x^{2}+y^{2}\right)^{3 / 4}(x+y)} \exp \left[-\frac{1}{2}(x+y)-\frac{1}{\sqrt{2}} \sqrt{x^{2}+y^{2}}\right] \frac{1}{\sqrt{2 \pi}} \int_{-\infty}^{\Delta} e^{-u^{2} / 2} d u \\
\Delta=\frac{2^{1 / 4}}{\left(x^{2}+y^{2}\right)^{1 / 4}}\left[T-T_{*}(x, y)\right]
\end{gathered}
$$

(d) $\quad y=O(1) ; x, T \rightarrow \infty$ with $T<x / \sqrt{2}$

$$
P(x, y, T) \sim \exp \left[-\frac{x+y+T}{2}-\frac{x^{2}}{4 T}\right] \frac{\sqrt{2}}{\pi T} \frac{1}{x-\sqrt{2} T}\left(y+\frac{2 T}{x}\right)
$$

(e) $\quad y=O(1) ; x, T \rightarrow \infty$ with $T>x / \sqrt{2}$

$$
P(x, y, T) \sim \frac{2^{5 / 4}}{\sqrt{\pi}} \exp \left[-\left(\frac{1}{2}+\frac{1}{\sqrt{2}}\right) x-\frac{y}{2}\right] \frac{y+\sqrt{2}}{x^{3 / 2}}
$$

(f) $\quad y=O(1) ; x, T \rightarrow \infty$ with $T \approx x / \sqrt{2}($ more precisely $T-x / \sqrt{2}=O(\sqrt{x})$ )

$$
\begin{gathered}
P(x, y, T) \sim \frac{2^{5 / 4}}{\sqrt{\pi}} \frac{y+\sqrt{2}}{x^{3 / 2}} \exp \left[-\left(\frac{1}{2}+\frac{1}{\sqrt{2}}\right) x-\frac{y}{2}\right] \frac{1}{\sqrt{2 \pi}} \int_{-\infty}^{\Delta_{1}} e^{-u^{2} / 2} d u, \\
\Delta_{1}=\frac{2^{1 / 4}}{\sqrt{x}}\left(T-\frac{x}{\sqrt{2}}\right)
\end{gathered}
$$

(g) $\quad x=O(1) ; y, T \rightarrow \infty$

$$
\text { use }(d)-(f) \text { and the symmetry } P(x, y, T)=P(y, x, T) \text {; }
$$

(h) $\quad x, y=O(1) ; T \rightarrow \infty$

$$
P(x, y, T)-P_{e q}(x, y) \sim\left[\frac{\sqrt{2}}{\pi^{3 / 2}} \frac{x+y}{x^{2}+y^{2}}-\frac{1}{2 \sqrt{2 \pi}}\right] e^{-(x+y) / 2 e^{-T / 2}} \frac{T^{3 / 2}}{2}
$$

(i) $\quad T \rightarrow \infty, x^{2}+y^{2}=O(T)$ 


$$
\begin{aligned}
P(x, y, T)-P_{e q}(x, y) \sim e^{-(x+y) / 2} e^{-T / 2}\left[-\frac{J(x, y, T)}{2 \pi T^{3}}+\frac{J_{1}(x, y, T)}{\left.\sqrt{2} \pi^{3 / 2} T^{7 / 2}\right]}\right. & \int_{0}^{\infty}(x+u)(y+u) \exp \left[-\frac{(x+u)^{2}+(y+u)^{2}}{4 T}\right] d u \\
J & =-\int_{0}^{\infty} \int_{0}^{1}(x+u)(y+u)\left[\frac{(x+u)^{2}+(y+u)^{2}}{4 T \eta}-3\right] \\
J_{1} & \times \exp \left[-\frac{(x+u)^{2}+(y+u)^{2}}{4 T \eta}\right] \frac{1}{\eta^{4} \sqrt{1-\eta}} d \eta d u .
\end{aligned}
$$

In $(a)-(c)$ it is assumed that $x, y, T$ are simultaneously large and of the same order of magnitude. In parts $(h)$ and $(i), P_{e q}$ is the steady-state or equilibrium distribution, as given by (3.7) with $a=+1$. We also note that when $T \rightarrow \infty$ with $x, y=$ $O(\sqrt{T}), J$ and $J_{1}$ are both of order $O\left(T^{3 / 2}\right)$. However, as $\left(x^{2}+y^{2}\right) / T \rightarrow 0, J_{1}$ becomes asymptotically larger than $J$.

To discuss the asymptotic results, we refer to the case where $x, y, T$ are all large and of comparable magnitudes as "interior" asymptotics, as this corresponds to the interior of the $(x, y, T)$ space. When two of $(x, y, T)$ are large and the third is not, we call the resulting asymptotic expansions "face" asymptotics; when only one of $(x, y, T)$ is large, we call it "edge" asymptotics. Thus, $(a)-(c)$ refers to interior asymptotics, $(d)-(g)$ to face asymptotics, and $(h)$ and $(i)$ refer to edge asymptotics along the $T$-axis or edge. Along the face where $x, y \rightarrow \infty$ with $T$ fixed, we can easily show that $(a)$ remains valid. Along edges where $x \rightarrow \infty$ with $y, T$ fixed (resp. $y \rightarrow \infty$ with $x, T$ fixed) we can show that $(d)$ (resp. $(g)$ ) remains valid. Hence, it is necessary to give different expressions along only two of the three faces and one of the three edges.

Parts $(a)-(c)$ show that for each fixed, large $x$ and $y$, there is a critical time $T=T_{*}$ at which the process has reached its steady-state. For times $T<T_{*}$, transient effects are important and the leading term in the expansion of $P$ depends upon time, while for $T>T_{*}$, the leading term depends only upon $x$ and $y$. We refer to the cylindrical surface $T=T_{*}(x, y)$ as a "front"; as time increases, this surface moves outward and eventually covers the entire space. Such fronts were previously found in other, one-dimensional queueing models (see [18, 23]).

Part $(h)$ gives the standard "relaxation rate" approximation. Such asymptotics are discussed for single server queues in Cohen [5] and for two tandem $M / M / 1$ queues in Blanc [2]. Our analysis shows that for large times $T$, the behavior of $P$ is different according as $x, y=O(1)(\mathrm{cf} .(h)) ; x, y=O(\sqrt{T})(\mathrm{cf} .(i)) ;$ or $x, y=O(T)$ (cf. $(a)-(c))$. We also note that along the face $y=0$, the transition in the behavior of $P$ occurs at $T=x / \sqrt{2}$, which is where the cylindrical front intersects the plane $y=0$.

We next give analogous asymptotic results for the unstable cases, where $\rho>1$ or $\rho=1$.

Theorem 3: For $x_{0}=y_{0}=0$ and $\rho>1$, we set $a=-1$. Asymptotic expansions of $P(x, y, T)$ are as follows:

(a) $\quad x, y, T \rightarrow \infty$

$$
P(x, y, T) \sim L(x, y, T) \exp [\Phi(x, y, T)]
$$


(b) $y=O(1) ; x, T \rightarrow \infty, T$

$$
\Phi(x, y, T)=\frac{x+y-T}{2}-\frac{x^{2}+y^{2}}{4 T}=-\frac{1}{4 T}\left[(x-T)^{2}+(y-T)^{2}\right]
$$

$$
P(x, y, T) \sim \exp \left[\frac{x+y-T}{2}-\frac{x^{2}}{4 T}\right] \frac{\sqrt{2}}{\pi T} \frac{1}{x+\sqrt{2 T}}\left(y+\frac{2 T}{x}\right)
$$

(c) $\quad x=O(1) ; y, T \rightarrow \infty$

$$
P(x, y, T) \sim \exp \left[\frac{x+y-T}{2}-\frac{y^{2}}{4 T}\right] \frac{\sqrt{2}}{\pi T} \frac{1}{y+\sqrt{2} T}\left(x+\frac{2 T}{y}\right)
$$

(d) $\quad x, y=O(1) ; T \rightarrow \infty$

$$
P(x, y, T) \sim\left[\frac{\sqrt{2}}{\pi^{3 / 2}} \frac{x+y}{x^{2}+y^{2}}+\frac{1}{2 \sqrt{2} \pi}\right] e^{(x+y) / 2 \frac{e^{-T / 2}}{T^{3 / 2}}}
$$

(e) $\quad T \rightarrow \infty, x^{2}+y^{2}=O(T)$

$$
P(x, y, T) \sim e^{(x+y) / 2} e^{-T / 2}\left[\frac{J(x, y, T)}{2 \pi T^{3}}+\frac{J_{1}(x, y, T)}{\sqrt{2} \pi^{3 / 2} T^{7 / 2}}\right],
$$

where $J$ and $J_{1}$ are as in Theorem 2 , part $(i)$.

Theorem 4: For $x_{0}=y_{0}=0$ and $\rho=1$, we set $a=0$; the asymptotic expansions are as follows:

(a) $\quad x, y, T \rightarrow \infty$

$$
P(x, y, T) \sim \frac{\sqrt{2}}{\pi} \frac{x y}{T(x+y)} \frac{1}{\sqrt{x^{2}+y^{2}}} \exp \left[-\frac{x^{2}+y^{2}}{4 T}\right]
$$

(b) $y=O(1) ; x, T \rightarrow \infty$

$$
P(x, y, T) \sim \exp \left[-\frac{x^{2}}{4 T}\right] \frac{\sqrt{2}}{\pi T x}\left(y+\frac{2 T}{x}\right)
$$

(c) $\quad x=O(1) ; y, T \rightarrow \infty$

$$
P(x, y, T) \sim \exp \left[-\frac{y^{2}}{4 T}\right] \frac{\sqrt{2}}{\pi T y}\left(x+\frac{2 T}{y}\right)
$$

(d) $\quad x, y=O(1) ; T \rightarrow \infty$

(e) $T \rightarrow \infty, x^{2}+y^{2}=O(T)$

$$
P(x, y, T) \sim \frac{\sqrt{2}}{\pi^{3 / 2} \sqrt{T}} \frac{x+y}{x^{2}+y^{2}}
$$

$$
P(x, y, T)=\frac{\sqrt{2}}{4 \pi^{3 / 2} T^{5 / 2}} \int_{0}^{\infty} \int_{0}^{1} \frac{(x+u)(y+u)}{\eta^{3} \sqrt{1-\eta}} \exp \left[-\frac{(x+u)^{2}+(y+u)^{2}}{4 T \eta}\right] d \eta d u
$$

We observe that if $\rho \geq 1, P \rightarrow 0$ as $T \rightarrow \infty$ for any fixed $x, y$. The expression in Theorem 4, part $(e)$ is in fact the exact result for $P=P_{I I}$ when $a=0$; for $T \rightarrow \infty$ with $x, y=O(\sqrt{T})$, this cannot be simplified any further.

If we specialize the result in Theorem $3(a)$ to $x-T=O(\sqrt{T})$ and $y-T=$ 
$O(\sqrt{T})$, we obtain

$$
L \exp \Phi \sim \frac{1}{4 \pi T} \exp \left[-\frac{\left(x^{\prime}\right)^{2}}{4 T}-\frac{\left(y^{\prime}\right)^{2}}{4 T}\right] ; x^{\prime}=x-T, y^{\prime}=y-T .
$$

This is similar to the diffusion approximation for the standard $M / M / 1$ queue for $\rho>1$ and shows that the two queues decouple in this limit. However, it is important to note that Theorem $3(a)$ is more general than (3.10) as the former assumes that $x, y, T$ are large, but $x / T$ and $y / T$ are not necessarily close to one. The coupling of the two queues is evident by the form of the function $L(x, y, T)$. The results in part $(d)$ again give the standard relaxation rate asymptotics.

Next we give analogous results for non-zero initial conditions $\left(x_{0}, y_{0}\right)$.

Theorem 2*: For fixed conditions $x_{0}$ and $y_{0}$ and $\rho<1$, we set $a=+1$. Asymptotic expansions of $P(x, y, T)$ are as follows:

(a) $\quad x, y, T \rightarrow \infty$ with $T<T_{*}(x, y)=\sqrt{\frac{x^{2}+y^{2}}{2}}$

$$
\begin{gathered}
P(x, y, T) \sim \exp [\Psi(x, y, T)]\left\{K(x, y, T) \exp \left[\frac{x_{0}+y_{0}}{2}\left(1-\frac{\sqrt{x^{2}+y^{2}}}{\sqrt{2} T}\right)\right]\right. \\
+\frac{1}{2 \pi T} e^{\left.\left(x_{0}+y_{0}\right) / 2\left[\sinh \left(\frac{x x_{0}+y y_{0}}{2 T}\right)+\frac{x-y}{x+y} \sinh \left(\frac{y y_{0}-x x_{0}}{2 T}\right)\right]\right\}}
\end{gathered}
$$

(b) $\quad x, y, T \rightarrow \infty$ with $T>T_{*}(x, y)$

$$
\text { same as Theorem 2, part (b) }
$$

(c) $\quad x, y, T \rightarrow \infty$ with $T \approx T_{*}(x, y)$

$$
\text { same as Theorem 2, part (c) }
$$

(d) $\quad y=O(1) ; x, T \rightarrow \infty$ with $T<x / \sqrt{2}$

$$
\begin{aligned}
P(x, y, T) & \sim \exp \left[-\frac{x+y+T}{2}-\frac{x^{2}}{4 T}\right]\left\{\frac{\sqrt{2}}{\pi T} \frac{1}{x-\sqrt{2} T}\left(y+\frac{2 T}{x}\right) \exp \left[\frac{x_{0}+y_{0}}{2}\left(1-\frac{x}{\sqrt{2} T}\right)\right]\right. \\
& \left.+\frac{1}{2 \pi T} e^{\left(x_{0}+y_{0}\right) / 2}\left[\left(\frac{4 T}{x^{2}}+\frac{2 y}{x}\right) \sinh \left(\frac{x x_{0}}{2 T}\right)+\left(\frac{y y_{0}}{T}+\frac{2 y_{0}}{x}\right) \cosh \left(\frac{x x_{0}}{2 T}\right)\right]\right\}
\end{aligned}
$$

(e) $\quad y=O(1) ; x, T \rightarrow \infty$ with $T>x / \sqrt{2}$

$$
\text { same as Theorem 2, part }(e)
$$

(f) $\quad y=O(1) ; x, T \rightarrow \infty$ with $T \approx x / \sqrt{2}$

$$
\text { same as Theorem } 2 \text {, part }(f)
$$

(g) $\quad x=O(1) ; y, T \rightarrow \infty$

use $(d)-(f)$ and the symmetry $P\left(x, y, T ; x_{0}, y_{0}\right)=P\left(y, x, T ; y_{0}, x_{0}\right)$ 
(h) $\quad x, y=O(1) ; T \rightarrow \infty$

$$
\begin{aligned}
P(x, y, T) & -P_{e q}(x, y) \\
& \sim e^{\left(x_{0}+y_{0}\right) / 2}\left(1-\frac{x_{0}+y_{0}}{2}\right)\left[\frac{\sqrt{2}}{\pi^{3 / 2}} \frac{x+y}{x^{2}+y^{2}}-\frac{1}{2 \sqrt{2} \pi}\right] e^{-(x+y) / 2 e^{-T / 2}} \frac{T^{3 / 2}}{O(T)}
\end{aligned}
$$

(i) $\quad T \rightarrow \infty, x^{2}+y^{2}=O(T)$

$$
\begin{gathered}
P(x, y, T)-P_{e q}(x, y) \\
\sim e^{-(x+y) / 2} e^{\left(x_{0}+y_{0}\right) / 2} e^{-T / 2}\left\{\frac{-J}{2 \pi T^{3}}+\left(1-\frac{x_{0}+y_{0}}{2}\right) \frac{J_{1}}{\sqrt{2} \pi^{3 / 2} T^{7 / 2}}\right. \\
\left.+\frac{x_{0}+y_{0}}{4 \pi T^{2}} J_{2}+\frac{x-y}{8 \pi T^{3}} J_{3}+\frac{x x_{0}+y y_{0}}{4 \pi T^{2}} \exp \left[-\frac{x^{2}+y^{2}}{4 T}\right]\right\} \\
J_{2}=\int_{0}^{\infty} \exp \left[-\frac{(x+u)^{2}+(y+u)^{2}}{4 T}\right] d u, \\
J_{3}=\int_{0}^{\infty}\left[y_{0}(y+u)-x_{0}(x+u)\right] \exp \left[-\frac{(x+u)^{2}+(y+u)^{2}}{4 T}\right] d u .
\end{gathered}
$$

Here $\Psi, K, P_{e q}, J$ and $J_{1}$ are as in Theorem 2. We note that when $x, y, T$ are all large, the dependence of $P$ on the initial conditions disappears as time increases past $T_{*}(x, y)$. For $T \rightarrow \infty$ with $x, y=O(\sqrt{T})$, we have $J_{2}=O(\sqrt{T})$ and $J_{3}=O(T)$.

Theorem 3*: For fixed initial conditions $x_{0}$ and $y_{0}$ and $\rho>1$, we set $a=-1$. Asymptotic expansions of $P(x, y, T)$ are as follows:

(a) $\quad x, y, T \rightarrow \infty$

$$
\begin{gathered}
P(x, y, T) \sim \exp [\Phi(x, y, T)]\left\{L(x, y, T) \exp \left[-\frac{x_{0}+y_{0}}{2}\left(1+\frac{\sqrt{x^{2}+y^{2}}}{\sqrt{2} T}\right)\right]\right. \\
+\frac{1}{\pi T(x+y)} e^{\left.-\left(x_{0}+y_{0}\right) / 2\left[x \cosh \left(\frac{x x_{0}}{2 T}\right) \sinh \left(\frac{y y_{0}}{2 T}\right)+y \sinh \left(\frac{x x_{0}}{2 T}\right) \cosh \left(\frac{y y_{0}}{2 T}\right)\right]\right\}}
\end{gathered}
$$

(b) $y=O(1) ; x, T \rightarrow \infty$

$$
\begin{gathered}
P(x, y, T) \sim \exp \left[\frac{x+y-T}{2}-\frac{x^{2}}{4 T}-\frac{x_{0}+y_{0}}{2}\right] \frac{1}{\pi T}\left(y+\frac{2 T}{x}\right) \\
\times\left\{\frac{\sqrt{2}}{x+\sqrt{2} T} \exp \left[-\frac{\left(x_{0}+y_{0}\right) x}{2 \sqrt{2} T}\right]+\frac{1}{x} \sinh \left(\frac{x x_{0}}{2 T}\right)+\frac{y_{0}}{2 T} \cosh \left(\frac{x x_{0}}{2 T}\right)\right\}
\end{gathered}
$$

(c) $\quad x=O(1) ; y, T \rightarrow \infty$

$$
\text { use }(b) \text { and the symmetry } P\left(x, y, T ; x_{0}, y_{0}\right)=P\left(y, x, T ; y_{0}, x_{0}\right)
$$

(d) $\quad x, y=O(1) ; T \rightarrow \infty$ 


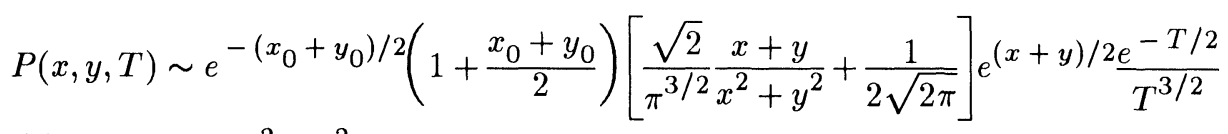

(e) $T \rightarrow \infty ; x^{2}+y^{2}=O(T)$

$$
\begin{aligned}
P(x, y, T) \sim & e^{(x+y) / 2} e^{-\left(x_{0}+y_{0}\right) / 2} e^{-T / 2}\left\{\frac{J}{2 \pi T^{3}}+\left(1+\frac{x_{0}+y_{0}}{2}\right) \frac{J_{1}}{\sqrt{2} \pi^{3 / 2} T^{7 / 2}}\right. \\
& \left.+\frac{x_{0}+y_{0}}{4 \pi T^{2}} J_{2}+\frac{x-y}{8 \pi T^{3}} J_{3}+\frac{x x_{0}+y y_{0}}{4 \pi T^{2}} \exp \left[-\frac{x^{2}+y^{2}}{4 T}\right]\right\} .
\end{aligned}
$$

Theorem 4*: For fixed $x_{0}$ and $y_{0}$ and $\rho=1$, we set $a=0$; the asymptotic expansions for $P$ are as follows:

(a) $\quad x, y, T \rightarrow \infty$

$$
\begin{aligned}
P(x, y, T) & \sim \frac{1}{\pi T} \exp \left[-\frac{x^{2}+y^{2}}{4 T}\right]\left\{\frac{\sqrt{2} x y}{(x+y) \sqrt{x^{2}+y^{2}}} \exp \left[-\frac{x_{0}+y_{0}}{2 \sqrt{2} T} \sqrt{x^{2}+y^{2}}\right]\right. \\
+ & \left.\frac{1}{x+y}\left[x \cosh \left(\frac{x x_{0}}{2 T}\right) \sinh \left(\frac{y y_{0}}{2 T}\right)+y \sinh \left(\frac{x x_{0}}{2 T}\right) \cosh \left(\frac{y y_{0}}{2 T}\right)\right]\right\}
\end{aligned}
$$

(b) $y=O(1) ;(x, T \rightarrow \infty)$

$$
P(x, y, T) \sim \frac{1}{\pi T} \exp \left[-\frac{x^{2}}{4 T}\right]\left(y+\frac{2 T}{x}\right)\left[\frac{\sqrt{2}}{x}+\frac{1}{x} \sinh \left(\frac{x x_{0}}{2 T}\right)+\frac{y_{0}}{2 T} \cosh \left(\frac{x x_{0}}{2 T}\right)\right]
$$

(c) $\quad x=O(1) ; y, T \rightarrow \infty$

$$
\text { use }(b) \text { and the symmetry } P\left(x, y, T ; x_{0}, y_{0}\right)=P\left(y, x, T ; y_{0}, x_{0}\right)
$$

(d) $\quad x, y=O(1) ; T \rightarrow \infty$

$$
\text { same as Theorem 4, part }(d)
$$

(e) $\quad T \rightarrow \infty, x^{2}+y^{2}=O(T)$

$$
\begin{gathered}
P(x, y, T) \sim \frac{x x_{0}+y y_{0}}{4 \pi T^{2}} \exp \left[-\frac{x^{2}+y^{2}}{4 T}\right]+\frac{x_{0}+y_{0}}{4 \pi T^{2}} J_{2}+\frac{x-y}{8 \pi T^{3}} J_{3}-\frac{x_{0}+y_{0}}{4 \pi T^{3}} J \\
+\frac{\sqrt{2}}{4 \pi^{3 / 2} T^{5 / 2}} \int_{0}^{\infty} \int_{0}^{1} \frac{(x+u)(y+u)}{\eta^{3} \sqrt{1-\eta}} \exp \left[-\frac{(x+u)^{2}+(y+u)^{2}}{4 T \eta}\right] d \eta d u .
\end{gathered}
$$

We note that when Theorem $3^{*}(a)$ is specialized to the scaling $x-T=O(\sqrt{T})$ and $y-T=O(\sqrt{T})$, we again obtain the decoupled Gaussian form (3.10), and the dependence on the initial conditions disappears in this limit. It is also possible to show that the double integral $\int_{0}^{\infty} \int_{0}^{\infty}\left[\right.$ expression in Theorem $\left.4^{*}(e)\right] d x d y=1$, which shows that when $\rho=1(a=0)$, the density is concentrated in the range $x, y=$ $O(\sqrt{T})$, as we might expect. For $\rho>1$, Theorem $3^{*}(a)$ shows that the density is concentrated where $x-T, y-T=O(\sqrt{T})$. For $\rho<1$, it is concentrated for $x, y=$ $O(1)$, and we can easily show that the double integral of the right-hand side of Theorem $2^{*}(h)$ vanishes.

All our asymptotic results apply for some or all of $x, y, T$ large but with fixed 
initial conditions $x_{0}, y_{0}$. Other interesting insights may be seen by evaluating $P\left(x, y, T ; x_{0}, y_{0}\right)$ for large initial conditions (i.e. $x_{0}$ and $y_{0}$ large), but we do not consider these here. Experience with other models (see $[18,23]$ ) shows that asymptotics for large initial conditions better reveal how the two-dimensional process $(X(T), Y(T))$ interacts with the barriers (reflecting boundaries) at $x=0$ and $y=0$.

\section{Derivation of the Integral Representations}

We obtain the representations for $P(x, y, T)$ in Theorem 1 by solving (2.10)-(2.14). We write the solution as $P=P_{I}+P_{I I}$ where $P_{I}$ will satisfy $(2.10)-(2.13)$ and be finite at the origin $(x, y)=(0,0)$. Hence $P_{I}$ is the Green's function for the parabolic PDE (2.10) with the BC (2.11) and (2.12). The second part $P_{I I}$ will satisfy (2.10)(2.12) and homogeneous initial conditions (i.e., $\left.P_{I I}(x, y, 0)=0\right)$. To uniquely determine $P_{I I}$, we will use the normalization condition (2.14).

First we set

$$
P_{I}(x, y, T)=e^{-a\left(x-x_{0}\right) / 2} e^{-a\left(y-y_{0}\right) / 2} R(x, y, T)
$$

to find that $R$ satisfies

$$
\begin{gathered}
R_{T}=\Delta R-\frac{a^{2}}{2} R, R_{x}+R_{y}=0 \text { when } x=0 \text { or } y=0 \\
\left.R\right|_{T=0}=\delta\left(x-x_{0}\right) \delta\left(y-y_{0}\right)
\end{gathered}
$$

where $\Delta=\partial_{x}^{2}+\partial_{y}^{2}$ is the Laplacian. Introducing the Laplace transform $\widehat{R}(x, y, T)=$ $\int_{0}^{\infty} e^{-\theta T} R(x, y, T) d T$, setting $\widehat{Q}=\left(\partial_{x}+\partial_{y}\right) \widehat{R}$ and noting that

$$
-\left(\partial_{x}+\partial_{y}\right) \delta\left(x-x_{0}\right) \delta\left(y-y_{0}\right)=\left(\partial_{x_{0}}+\partial_{y_{0}}\right) \delta\left(x-x_{0}\right) \delta\left(y-y_{0}\right)
$$

we find that $\widehat{Q}=\left(\partial_{x_{0}}+\partial_{y_{0}}\right) \widetilde{Q}$ where $\widetilde{Q}$ satisfies

$$
\left[\Delta-\left(\theta+\frac{a^{2}}{2}\right)\right] \widetilde{Q}=\delta\left(x-x_{0}\right) \delta\left(y-y_{0}\right), \widetilde{Q}=0 \text { when } x=0 \text { or } y=0 \text {. }
$$

This is a standard Green's function problem for the modified Helmholtz operator with Dirichlet boundary conditions. The solution of (4.3) is easily obtained using the method of images, and we have

$$
\begin{gathered}
\tilde{Q}=-\frac{1}{2 \pi}\left\{K_{0}\left(\sqrt{\theta+\frac{a^{2}}{2}} \sqrt{\left(x-x_{0}\right)^{2}+\left(y-y_{0}\right)^{2}}\right)\right. \\
+K_{0}\left(\sqrt{\theta+\frac{a^{2}}{2}} \sqrt{\left(x+x_{0}\right)^{2}+\left(y+y_{0}\right)^{2}}\right)-K_{0}\left(\sqrt{\theta+\frac{a^{2}}{2}} \sqrt{\left(x+x_{0}\right)^{2}+\left(y-y_{0}\right)^{2}}\right) \\
\left.-K_{0}\left(\sqrt{\theta+\frac{a^{2}}{2}} \sqrt{\left(x-x_{0}\right)^{2}+\left(y+y_{0}\right)^{2}}\right)\right\} .
\end{gathered}
$$

Using (4.4) we compute $\widehat{Q}=\left(\partial_{x_{0}}+\partial_{y_{0}}\right) \widetilde{Q}$ and then $\widehat{R}$ from $\widehat{R}=\left(\partial_{x}+\partial_{y}\right)^{-1} \widehat{Q}$, where the operator $\left(\partial_{x}+\partial_{y}\right)^{-1}$ is defined by 


$$
\begin{gathered}
\left(\partial_{x}+\partial_{y}\right)^{-1} F(x, y)=-\int_{(x+y) / 2}^{\infty} F\left(u+\frac{x-y}{2}, u+\frac{y-x}{2}\right) d u \\
=-\int_{0}^{\infty} F(u+x, u+y) d u
\end{gathered}
$$

We denote by $\mathscr{L}$ and $\mathcal{L}^{-1}$ the Laplace transform operator and its inverse, respectively. Hence,

$$
\mathcal{L}[F(T)]=\widehat{F}(\theta)=\int_{0}^{\infty} e^{-\theta T} F(T) d T, F(T)=\mathcal{L}^{-1}[\widehat{F}(\theta)]=\frac{1}{2 \pi i} \int_{\mathrm{Br}} e^{\theta T} \widehat{F}(\theta) d \theta
$$

where $\mathrm{Br}$ is an appropriate Bromwich contour in the complex $\theta$-plane. From the tables of the Laplace transforms, we obtain

$$
\mathcal{L}^{-1}\left[K_{0}\left(k \sqrt{\theta+\frac{a^{2}}{2}}\right)\right]=\frac{1}{2 T} \exp \left[-\frac{a^{2}}{2} T-\frac{k^{2}}{4 T}\right], k>0
$$

where $k$ is any constant. Using (4.5) and (4.4) we obtain the solution to (4.2) in the form

$$
\begin{gathered}
R(x, y, T)=-\frac{1}{4 \pi T} e^{-a^{2} T / 2}\left(\partial_{x}+\partial_{y}\right)^{-1}\left(\partial_{x_{0}}+\partial_{y_{0}}\right)\left\{\exp \left[-\frac{\left(x-x_{0}\right)^{2}+\left(y-y_{0}\right)^{2}}{4 T}\right]\right. \\
+\exp \left[-\frac{\left(x+x_{0}\right)^{2}+\left(y+y_{0}\right)^{2}}{4 T}\right]-\exp \left[-\frac{\left(x+x_{0}\right)^{2}+\left(y-y_{0}\right)^{2}}{4 T}\right] \\
\left.-\exp \left[-\frac{\left(x-x_{0}\right)^{2}+\left(y+y_{0}\right)^{2}}{4 T}\right]\right\}
\end{gathered}
$$

and then $P_{I}$ is given by (4.1). A straightforward calculation shows

$$
\begin{gathered}
-\left(\partial_{x}+\partial_{y}\right)^{-1}\left(\partial_{x_{0}}+\partial_{y_{0}}\right) \exp \left[-\frac{1}{4 T}\left(\left(x \mp x_{0}\right)^{2}+\left(y \mp y_{0}\right)^{2}\right)\right] \\
= \pm \exp \left[-\frac{1}{4 T}\left(\left(x \mp x_{0}\right)^{2}+\left(y \mp y_{0}\right)^{2}\right)\right]
\end{gathered}
$$

and

$$
\begin{gathered}
-\left(\partial_{x}+\partial_{y}\right)^{-1}\left(\partial_{x_{0}}+\partial_{y_{0}}\right)\left\{-\exp \left[-\frac{1}{4 T}\left(\left(x+x_{0}\right)^{2}+\left(y-y_{0}\right)^{2}\right)\right]\right\} \\
=\frac{1}{2 T}\left(x+x_{0}-y+y_{0}\right) \int_{0}^{\infty} \exp \left[-\frac{1}{4 T}\left(\left(x+u+x_{0}\right)^{2}+\left(y+u-y_{0}\right)^{2}\right)\right] d u .
\end{gathered}
$$

The use of (4.6) in (4.1) then gives precisely $P_{I}$ in Theorem 1.

We note that $P_{I} \rightarrow 0$ as $T \rightarrow \infty$ and thus (4.1) cannot represent the full density of the diffusion model, especially when $\rho<1$. The solution (4.4) to (4.3) is a particular solution to the Green's function problem. However, we now show that there also exists a non-zero solution to the corresponding homogeneous problem (obtained by 
dropping the delta function in (4.3)) that plays a role in ultimately determining $P(x, y, T)$.

We return to (2.10)-(2.12) and set

$$
P_{I I}(x, y, T)=e^{-a(x+y) / 2} e^{-a^{2} T / 2} U(x, y, T)
$$

to find that $U$ satisfies the problem

$$
\Delta U=U_{T}, U_{x}+U_{y}=0 \text { when } x=0 \text { or } y=0, U=0 \text { when } T=0 .
$$

To solve (4.8) we introduce the Laplace transform $\widetilde{U}(x, y ; s)=\int_{0}^{\infty} e^{-s T} U(x, y, T) d T$ and set $V=\widetilde{U}_{x}+\tilde{U}_{y}$. The function $V=V(x, y ; s)$ satisfies the modified Helmholtz equation $(\Delta-s) V=0$ with the boundary conditions $V(x, 0 ; s)=V(0, y ; s)=0$. The only solution of this problem that is integrable both as $\rho=\sqrt{x^{2}+y^{2}} \rightarrow \infty$ and as $\rho \rightarrow 0$ is given by

$$
V(x, y ; s)=\bar{F}(s) \frac{2 x y}{x^{2}+y^{2}} K_{2}\left(\sqrt{s} \sqrt{x^{2}+y^{2}}\right)
$$

where $K_{2}(\cdot)$ is the Bessel function. By using integral representations for Bessel functions we also have

$$
\frac{2 x y}{x^{2}+y^{2}} K_{2}\left(\sqrt{s} \sqrt{x^{2}+y^{2}}\right)=\frac{i}{2} \int_{-\infty}^{\infty} \sinh (2 \eta) e^{-\sqrt{s} x \cosh \eta} e^{-i \sqrt{s} y \sinh \eta} d \eta
$$

and this holds true for $x>0$ and $y \geq 0$.

Using the representation (4.10) in (4.9) we obtain

$$
\begin{aligned}
& \widetilde{U}(x, y ; s)=\left(\partial_{x}+\partial_{y}\right)^{-1} V=-\int_{0}^{\infty} V(x+u, y+u) d u \\
& =F(s) \int_{-\infty}^{\infty} \frac{i \sinh (2 \eta)}{\cosh +i \sinh \eta} e^{-\sqrt{s} x \cosh \eta} e^{-i \sqrt{s} y \sinh \eta} d \eta
\end{aligned}
$$

where $F(s)=-\bar{F}(s) /(2 \sqrt{s})$. Finally, we obtain the solution $U$ to (4.8) by inverting the Laplace transform in (4.11). Then $P_{I I}$ is given by (4.7). It remains to determine the function $F(\cdot)$. In view of $(4.7)-(4.11), \widehat{P}_{I I}=\mathcal{L}\left[P_{I I}\right](\theta)$ is

$$
\widehat{P}_{I I}=e^{-a(x+y) / 2} F \int_{-\infty}^{\infty} \frac{i \sinh (2 \eta)}{\cosh \eta+i \sinh \eta} e^{-\sqrt{\theta+a^{2} / 2}(x \cosh \eta+i y \sinh \eta)} d \eta
$$

We use (2.14), which in terms of Laplace transforms is

$$
\int_{0}^{\infty} \int_{0}^{\infty} \widehat{P}_{I}(x, y ; \theta) d x d y+\int_{0}^{\infty} \int_{0}^{\infty} \widehat{P}_{I I}(x, y ; \theta) d x d y=\frac{1}{\theta}
$$


By noting that $P_{I}$ satisfies (2.10) and this function is finite at the origin $x=y=0$, we integrate $(2.10)$ over the first quadrant and obtain

$$
\begin{aligned}
& \frac{d}{d T} \int_{0}^{\infty} \int_{0}^{\infty} P_{I} d x d y=\int_{0}^{\infty}-\left[P_{I, x}(0, y, T)+a P_{I}(0, y, T)\right] d y \\
& +\int_{0}^{\infty}-\left[P_{I, y}(x, 0, T)+a P_{I}(x, 0, T)\right] d x \\
& =\int_{0}^{\infty} P_{I, y}(0, y, T) d y+\int_{0}^{\infty} P_{I, x}(x, 0, T) d x=-2 P_{I}(0,0, T) .
\end{aligned}
$$

Here we have used the boundary conditions (2.11) and (2.12). Using Theorem 1 to evaluate $P_{I}(0,0, T)$ in $(4.14)$ we are led to

$$
-\frac{1}{2} \frac{d}{d T} \int_{0}^{\infty} \int_{0}^{\infty} P_{I}(x, y, T) d x d y=\frac{x_{0}+y_{0}}{4 \sqrt{2 \pi} T^{3 / 2}} \exp \left[-\frac{1}{2}\left(a \sqrt{T}-\frac{x_{0}+y_{0}}{2 \sqrt{T}}\right)^{2}\right]
$$
Taking the Laplace transform of (4.15) and noting that $\int_{0}^{\infty} \int_{0}^{\infty} P_{I}(x, y, 0) d x d y=1$ we
obtain

$$
\int_{0}^{\infty} \int_{0}^{\infty} \widehat{P}_{I}(x, y ; \theta) d x d y=\frac{1}{\theta}\left\{1-\exp \left[\left(x_{0}+y_{0}\right)\left(\frac{a}{2}-\sqrt{\frac{\theta}{2}+\frac{a^{2}}{4}}\right)\right]\right\} \text {. }
$$

Next taking $a>0$ and integrating (4.12) over the first quadrant, we arrive at

$$
\begin{aligned}
& \int_{0}^{\infty} \int_{0}^{\infty} \widehat{P}_{I I}(x, y ; \theta) d x d y \\
& \quad=F(s) i \int_{-\infty}^{\infty} \frac{\sinh (2 \eta)}{\cosh \eta+i \sinh \eta} \frac{4}{(a+2 \sqrt{s} \cosh \eta)(a+2 i \sqrt{s} \sinh \eta)} d \eta
\end{aligned}
$$

where $s=\theta+a^{2} / 2$. The last integral is readily evaluated using residues. Its integrand has simple poles at $\eta=i \pi / 4,5 i \pi / 4$ and at $\eta=i u_{0}$ where $u_{0}$ is the solution to $\sin \left(u_{0}\right)=a /(2 \sqrt{s})=a / \sqrt{4 \theta+2 a^{2}}$ in the range $0<u_{0}<\pi / 2$ (recall that $a>0$ ). Let $I_{0}$ denote the integral in (4.17), which goes from $-\infty$ to $+\infty$ in the $\eta$-plane and let $I_{1}$ denote the integral over a shifted contour that goes from $-\infty+i \pi / 2$ to $+\infty+$ $i \pi / 2$. The only poles inside the strip $0 \leq \operatorname{Im}(\eta) \leq \pi / 2$ are at $\eta=i \pi / 4$ and $\eta=i u_{0}$. Hence

$$
I_{0}-I_{1}=2 \pi i\left[\operatorname{res}\left(\eta=\frac{i \pi}{4}\right)+\operatorname{res}\left(\eta=i u_{0}\right)\right] \text {. }
$$

By letting $\eta \rightarrow-u+i \pi / 2$ we see that $I_{1}=-I_{0}$. Then explicitly computing the residues in (4.18) we find from (4.17) that

$$
\int_{0}^{\infty} \int_{0}^{\infty} \widehat{P}_{I I}(x, y ; \theta) d x d y=F(s) \frac{\pi}{\theta} \frac{a-\sqrt{a^{2}+2 \theta}}{\sqrt{\theta+a^{2} / 2}}
$$


In view of (4.13), (4.16) and (4.19), we determine $F$ as

$$
F=\frac{\sqrt{a^{2}+2 \theta}+a}{2 \pi \theta} \sqrt{\theta+\frac{a^{2}}{2}} \exp \left[\left(x_{0}+y_{0}\right)\left(\frac{a}{2}-\sqrt{\frac{\theta}{2}+\frac{a^{2}}{4}}\right)\right] .
$$

In obtaining (4.20) we have assumed that $a>0$. However, an analogous calculation shows that in fact the same result is true for $a \leq 0$. With (4.20) and (4.12) we have now completely determined $\widehat{P}_{I I}$ and hence the final result for the density $P(x, y, T)$ is $P=\mathcal{L}^{-1}\left[\widehat{P}_{I}+\widehat{P}_{I I}\right]=P_{I}+\mathcal{L}^{-1}\left[\widehat{P}_{I I}\right]$. We have thus established the representation in Theorem 1 arising from (3.1), (3.4) and (3.6).

We now derive the alternate representations (3.3) and (3.5) for $P_{I I}$. To obtain (3.5) we use the identity

$$
\frac{1}{\cosh \eta+i \sinh \eta}=\sqrt{s} \int_{0}^{\infty} e^{-\sqrt{s}(\cosh \eta+i \sinh \eta) u} d u
$$

to express $\widehat{P}_{I I}$ in (4.12) as a double integral. However, the integral over $\eta$ (with (4.21)) may be explicitly evaluated by shifting the contour with $\eta=z+i \omega$, where $\omega=\omega(x, y, u)$ is chosen to satisfy

Hence,

$$
\cos (\omega)=\frac{x+u}{\sqrt{(x+u)^{2}+(y+u)^{2}}}, \sin (\omega)=\frac{-(y+u)}{\sqrt{(x+u)^{2}+(y+u)^{2}}} .
$$

$$
\begin{gathered}
e^{a(x+y) / 2} \widehat{P}_{I I} / F=\sqrt{s} \int_{0}^{\infty} \int_{-\infty}^{\infty} i \sinh [2(z+i \omega)] e^{-\sqrt{s} \sqrt{(x+u)^{2}+(y+u)^{2}} \cosh z} d z d u \\
=\sqrt{s} \int_{0}^{\infty}(-\sinh (2 \omega)) \int_{-\infty}^{\infty} \cosh (2 z) e^{-\sqrt{s} \sqrt{(x+u)^{2}+(y+u)^{2}} \cosh z} d z d u \\
=-4 \int_{0}^{\infty} \sin \omega \cos \omega \sqrt{s} K_{2}\left(\sqrt{s} \sqrt{(x+u)^{2}+(y+u)^{2}}\right) d u
\end{gathered}
$$

where $s=\theta+a^{2} / 2$. Using (4.20) and expressing $\sin \omega \cos \omega$ in terms of $x, y$ and $u$, this establishes (3.5).

To obtain (3.3), we invert the transform in (3.5). We use

in (3.5) to get

$$
K_{2}(z)=\frac{1}{2} z^{2} \int_{0}^{\infty} t^{-3} e^{-t / 2} \exp \left(-\frac{z^{2}}{2 t}\right) d t
$$

$$
\begin{aligned}
\widehat{P}_{I I}= & \frac{\sqrt{2}}{\pi \theta}\left(s^{3 / 2}+\frac{a}{\sqrt{2}} s\right) e^{a\left(x_{0}+y_{0}-x-y\right) / 2} \int_{0}^{\infty} \int_{0}^{\infty}(x+u)(y+u) t^{-3} \\
& \times \exp \left[-\sqrt{s}\left(\frac{x_{0}+y_{0}}{\sqrt{2}}+\frac{t}{2}+\frac{(x+u)^{2}+(y+u)^{2}}{2 t}\right)\right] d t d u
\end{aligned}
$$


Then using the inverse transforms

$$
\mathcal{L}^{-1}\left[\frac{1}{\theta} G(\theta)\right]=\int_{0}^{T} \mathcal{L}^{-1}[G(\theta)](\xi) d \xi, \quad \mathcal{L}^{-1}\left[s^{\frac{n-1}{2}} e^{-k \sqrt{s}]}=\frac{e^{-k^{2} / 4 T}}{2^{n} \sqrt{\pi} T^{\frac{n+1}{2}}} H_{n}\left(\frac{k}{2 \sqrt{T}}\right)\right.
$$

where $H_{n}(\cdot)$ is the $n$th Hermite polynomial, we ultimate obtain (3.3) from (4.23). This completes the analysis.

\section{Asymptotic Expansions}

We establish Theorems $2-4$ (and also $2^{*}-4^{*}$ ) by evaluating Theorem 1 in various asymptotic limits. We do not give all the technical details, but simply sketch the main ideas.

We first consider $\rho<1$ and set $a=+1$ in (3.5). For any fixed $\theta$ with $\operatorname{Re}(\theta)>$ $-1 / 2$ and $x$ and/or $y$ large, we can approximate the Bessel function $K_{2}(\cdot)$ by its asymptotic expansion:

$$
K_{2}(z)=e^{-z} \sqrt{\frac{\pi}{2 z}}\left[1+\frac{15}{8 z}+O\left(z^{-2}\right)\right], \quad z \rightarrow \infty .
$$

Using the leading term in (5.1) in (3.5), we evaluate the integral over $u$ asymptotically for $x^{2}+y^{2} \rightarrow \infty$. The major contribution to the integral in (3.5) will come from the lower limit $u=0$, and Watson's lemma [3] then yields

$$
\begin{aligned}
\mathrm{JN} & \equiv \int_{0}^{\infty} \frac{(x+u)(y+u)}{(x+u)^{2}+(y+u)^{2}} K_{2}\left(\sqrt{\theta+\frac{1}{2}} \sqrt{(x+u)^{2}+(y+u)^{2}}\right) d u \\
& \sim \frac{x y}{\left(x^{2}+y^{2}\right)^{3 / 4}} \sqrt{\frac{\pi}{2}} \frac{1}{(x+y)(\theta+1 / 2)^{3 / 4}} \exp \left[-\sqrt{\theta+\frac{1}{2}} \sqrt{x^{2}+y^{2}}\right]
\end{aligned}
$$

The error term in (5.2) is order $O\left(\rho^{-1}\right)\left(\rho=\sqrt{x^{2}+y^{2}}\right)$ and it may be easily calculated by using the higher order terms in (5.1) and then carrying out a more precise evaluation of the $u$-integral in (3.5). We use (5.2) in (3.4) and set $\theta=\xi-1 / 2$ to get

$$
P_{I I} \sim \frac{x y e^{-T / 2}}{\left(x^{2}+y^{2}\right)^{3 / 4}} \frac{e^{-(x+y) / 2}}{x+y} \sqrt{\frac{2}{\pi}}\left\{\frac{1}{2 \pi i} \int_{\mathrm{Br}} \frac{2 \xi^{1 / 4}}{\sqrt{2 \xi}-1} e^{\xi T-\sqrt{\xi} \sqrt{x^{2}+y^{2}}} d \xi\right\}
$$

where $\operatorname{Re}(\xi)>1 / 2$ on the $\operatorname{Br}$ contour.

For $x, y$ and $T$ simultaneously large, we evaluate the integral in (5.3) by the saddle point (or steepest descent) method. There is a saddle point with

$$
\frac{\partial}{\partial \xi}\left[\xi T-\sqrt{\xi} \sqrt{x^{2}+y^{2}}\right]=T-\frac{1}{2 \sqrt{\xi}} \sqrt{x^{2}+y^{2}}=0,
$$

so that

$$
\xi=\xi_{0}(x, y, T) \equiv \frac{x^{2}+y^{2}}{4 T^{2}} .
$$

Thus the saddle lies on the positive real axis and we can easily show that the directions of steepest descent at $\xi_{0}$ are $\arg \left(\xi-\xi_{0}\right)= \pm \pi / 2$. From (5.3), we also note that the integrand has a simple pole at $\xi=1 / 2$ and a branch point at $\xi=0$. For 
$\xi_{0}(x, y, T)>1 / 2$, the saddle lies to the right of the pole and we may thus shift the $\mathrm{Br}$ contour into the steepest descent contour through $\xi_{0}$. From (5.5), we see that $\xi_{0}>1 / 2$ is equivalent to $T<\sqrt{\frac{x^{2}+y^{2}}{2}}=T_{*}$ and this establishes part $(a)$ of Theorem 2. We also note that

$$
T \xi_{0}-\sqrt{\xi_{0}} \sqrt{x^{2}+y^{2}}=-\frac{x^{2}+y^{2}}{4 T}, \frac{2 \xi_{0}}{\sqrt{2 \xi_{0}}-1}=\frac{x^{2}+y^{2}}{\sqrt{2} T} \frac{1}{\sqrt{x^{2}+y^{2}}-\sqrt{2} T} .
$$

When $T>T_{*}$, we have $\xi_{0}<1 / 2$. Thus shifting the contour in (5.3) into the steepest descent contour, we must take into account the contribution to the integral from the pole at $\xi=1 / 2$. We can easily show that the residue from this pole dominates the integral through the saddle point and hence

$$
\begin{gathered}
\mathrm{IN} \equiv \frac{1}{2 \pi i} \int_{\mathrm{Br}} \frac{2 \xi^{1 / 4}}{\sqrt{2 \xi}-1} e^{\xi T-\sqrt{\xi} \sqrt{x^{2}+y^{2}}} d \xi \sim\left[\text { residue at } \xi=\frac{1}{2}\right] \\
=2^{3 / 4} e^{T / 2} e^{-\sqrt{x^{2}+y^{2}} / \sqrt{2}} .
\end{gathered}
$$

Using the above in (5.3) we obtain part (b) of Theorem 2. The dependence on time disappears and the result is precisely the leading term in the asymptotic expansion of the equilibrium density $P_{e q}(x, y)$, for $x$ and $y$ large.

When $T \approx T_{*}$, the saddle point and pole are close to each other. Now we expand the integrand using $\xi=1 / 2$ and set

with which

$$
\xi-\frac{1}{2}=\frac{2^{1 / 4}}{\left(x^{2}+y^{2}\right)^{1 / 4}} z=O\left(\rho^{-1 / 2}\right)
$$

and hence

$$
\begin{gathered}
\xi T-\sqrt{\xi} \sqrt{x^{2}+y^{2}}=\frac{T}{2}+z\left[\frac{2^{1 / 4} T}{\left(x^{2}+y^{2}\right)^{1 / 4}}-\frac{\left(x^{2}+y^{2}\right)^{1 / 4}}{2^{1 / 4}}\right] \\
-\sqrt{\frac{x^{2}+y^{2}}{2}}+\frac{1}{2} z^{2}+o(1)
\end{gathered}
$$

$$
\mathrm{IN} \sim 2^{3 / 4} e^{T / 2} e^{-\sqrt{x^{2}+y^{2}} / \sqrt{2}} \frac{1}{2 \pi i} \int_{C} e^{\Delta z} e^{z^{2} / 2} \frac{d z}{z}, \Delta=\frac{2^{1 / 4}\left(T-T_{*}\right)}{\left(x^{2}+y^{2}\right)^{1 / 4}} .
$$

Here the contour $C$ goes along the imaginary axis in the $z$-plane, with an indentation to the right of $z=0$. The last integral is easily evaluated as $(2 \pi)^{-1 / 2} \int_{-\infty}^{\Delta} e^{-u^{2} / 2} d u$ and thus obtain part $(c)$ of Theorem 2 .

Next, we consider the face asymptotics where $y=O(1)$ and $x, T \rightarrow \infty$. For $x \rightarrow \infty$ and $y=O(1)$, we have $\left[(x+u)^{2}+(y+u)^{2}\right]^{1 / 2}=x+u+o(1)$. Then the leading term in the expansion of $\mathrm{JN}$ in (5.2) becomes

$$
\mathrm{JN} \sim \frac{1}{(\theta+1 / 2)^{3 / 4}} \sqrt{\frac{\pi}{2}} \frac{1}{x^{3 / 2}}\left[y+\frac{1}{\sqrt{\theta+1 / 2}}\right] \exp [-\sqrt{\theta+1 / 2} x] .
$$


Using (5.6) in (5.3) we again expand the $\xi$-integral using the saddle point method. The analog of $(5.4)$ is now $T-x /(2 \sqrt{\xi})=0$, so that the saddle point is at $\xi=\xi_{0}^{\prime} \equiv$ $x^{2} /\left(4 T^{2}\right)$. For $T<x / \sqrt{2}$ we have $\xi_{0}^{\prime}>1 / 2$ and this leads to Theorem $2(d)$. For $T>x / \sqrt{2}$, we have $\xi_{0}^{\prime}<1 / 2$. Then the pole at $\xi=1 / 2$ determines the asymptotic behavior of $P$ and we obtain Theorem $2(e)$. Theorem $2(f)$ applies to the case where $\xi_{0}^{\prime} \approx 1 / 2$, and the result is derived in a similar manner as for part $(c)$.

We examine the limit $T \rightarrow \infty$ with $x, y=o(T)$. First we fix $x$ and $y$. For $\left(x^{2}+y^{2}\right) / T^{2} \rightarrow 0$, the saddle point in (5.5) approaches the branch point at $\xi=0$ and hence the calculations that led to parts $(a)-(c)$ of Theorem 2 are no longer valid. For $T \rightarrow \infty$, the leading term for $P$ is clearly the steady state density $P_{e q}$. To obtain the approach to equilibrium we write

$$
P-P_{e q}=\frac{1}{2 \pi i} \int_{\mathrm{Br}^{\prime}} e^{\theta T} \widehat{P}_{I I} d \theta
$$

where on $\mathrm{Br}^{\prime},-1 / 2<\operatorname{Re}(\theta)<0$. For $T \rightarrow \infty$ the behavior of the integrand in (5.7) is determined by the branch point at $\theta=-1 / 2(\xi=0)$, which is the singularity with the largest real part. We write

$$
K_{2}(z)=\frac{2}{z^{2}}+\frac{2}{z^{2}} \int_{0}^{\infty} w e^{-w}\left[\exp \left(-\frac{z^{2}}{4 w}\right)-1\right] d w
$$

and note that $K_{2}(z) \sim 2 z^{-2}$ as $z \rightarrow 0$. We first compute the contribution to (5.7) (with (3.5)) from the first term in the right-hand side of (5.8), which gives

$$
e^{-(x+y) / 2} e^{-T / 2} \frac{1}{2 \pi i} \frac{2}{\pi} \int_{\mathrm{Br}^{\prime}} \frac{2 \xi}{\sqrt{2 \xi}-1} e^{\xi T} \int_{0}^{\infty} \frac{2(x+u)(y+u)}{\left[(x+u)^{2}+(y+u)^{2}\right]^{2} \xi} d u d \xi
$$

For $\xi$ small, we have $4 / \sqrt{(2 \xi)}-1=-4-4 \sqrt{2 \xi}+O(\xi)$ and the $u$-integral in (5.9) is easily evaluated as

$$
\int_{0}^{\infty} \frac{(x+u)(y+u)}{\left[(x+u)^{2}+(y+u)^{2}\right]^{2}} d u=\frac{1}{4} \frac{x+y}{x^{2}+y^{2}}, x^{2}+y^{2}>0
$$

Thus (5.9) becomes, for $T \rightarrow \infty$,

$$
\frac{\sqrt{2}}{\pi^{3 / 2}} \frac{x+y}{x^{2}+y^{2}} e^{-(x+y) / 2 e^{-T / 2}} \frac{T^{3 / 2}}{}
$$

To evaluate the contribution to (5.7) (with (3.5)) from the integral in (5.8) we use (5.8) in (5.7). Apart from the factor $e^{-(x+y+T) / 2}$, this gives (with $A^{2}=(x+$ $\left.u)^{2}+(y+u)^{2}\right)$

$$
\begin{gathered}
\frac{2}{\pi} \frac{1}{2 \pi i} \int_{\mathrm{Br}^{\prime}} \frac{2 \xi}{\sqrt{2 \xi}-1} e^{\xi T} \int_{0}^{\infty} \frac{(x+u)(y+u)}{(x+u)^{2}+(y+u)^{2}}\left[K_{2}\left(\sqrt{\xi} \sqrt{(x+u)^{2}+(y+u)^{2}}\right)\right. \\
\left.-\frac{2}{\xi(x+u)^{2}+(y+u)^{2}}\right] d u d \xi
\end{gathered}
$$




$$
\begin{gathered}
\sim \frac{2}{\pi} \int_{0}^{\infty} \frac{(x+u)(y+u)}{A^{2}} \frac{1}{2 \pi i} \int_{\mathrm{Br}^{\prime}}(-2 \xi) e^{\xi T} \int_{0}^{\infty} w e^{-w} \frac{2}{A^{2} \xi}\left[\exp \left(-\frac{A^{2} \xi}{4 w}\right)-1\right] d w d \xi d u \\
\left.=\frac{8}{\pi} \int_{0}^{\infty} \frac{(x+u)(y+u)}{A^{4}}\left[\int_{0}^{\infty} \frac{1}{2 \pi i} \int_{\mathrm{Br}^{\prime}} \int^{T \xi}-\exp \left[\left(T-\frac{A^{2}}{4 w}\right) \xi\right]\right\} d \xi\right] w e^{-w} d w d u \\
=\frac{8}{\pi} \int_{0}^{\infty} \frac{(x+u)(y+u)}{A^{4}} \int_{0}^{\infty} w e^{-w}\left[\delta(T)-\delta\left(T-\frac{A^{2}}{4 w}\right)\right] d w d u \\
=\frac{8}{\pi} \int_{0}^{\infty} \frac{(x+u)(y+u)}{A^{4}}(-1) \int_{0}^{\infty} w e^{-w} \frac{4}{A^{2}}\left(\frac{A^{2}}{4 T}\right)^{2} \delta\left(w-\frac{A^{2}}{4 T}\right) d w d u \\
=-\frac{1}{2 \pi T^{3}} \int_{0}^{\infty}(x+u)(y+u) \exp \left[-\frac{A^{4}}{4 T}\right] d u \\
\sim-\frac{1}{2 \pi T^{3}} \int_{0}^{\infty} u^{2} \exp \left[-\frac{u^{2}}{2 T}\right] d u(\operatorname{since} T \rightarrow \infty \text { with } x, y=O(1)) \\
=-\frac{1}{2 \sqrt{2 \pi}} \frac{1}{T^{3 / 2}} .
\end{gathered}
$$

Here we have set $\delta(T)=0$ (since $T \rightarrow \infty$ ) and used some standard identities involving delta functions. Upon multiplying the last expression in $(5.11)$ by $e^{-(x+y+T) / 2}$ and adding the result to $(5.10)$ we obtain part $(h)$ of Theorem 2 .

Now observe from (5.7) that for $x, y, T$ all large and of the same order, the leading term in the expansion of $P-P_{e q}$ is determined by the saddle point in (5.5). Thus $P-P_{e q}$ is given by the expression $K e^{\Psi}$ in Theorem $2(a)$ for this range of $x$ and $y$, and $\stackrel{e q}{T}>T_{*}$. However, $K e^{\Psi}$, when expanded for $x, y \rightarrow 0$, does not agree with the expression in Theorem $2(h)$. Thus, the two expansions do not "asymptotically match" and this indicates that another expansion(s) is needed for the range $T \rightarrow \infty$ with $x, y \rightarrow \infty$ but $x, y=o(T)$. To construct this "intermediate" expansion we return to (3.5) (with $a=+1$ and $x_{0}=y_{0}=0$ ), scale $\xi$ to be small, and use the integral representation

$$
K_{2}(z)=\frac{1}{2} z^{2} \int_{0}^{\infty} w^{-3} \exp \left[-\frac{1}{2}\left(w+\frac{z^{2}}{w}\right)\right] d w
$$

which follows from (5.8). We thus obtain

$$
\begin{gathered}
P-P_{e q}=e^{-(x+y+T) / 2} \frac{2}{\pi} \frac{1}{2 \pi i} \int_{\mathrm{Br}^{\prime}} \frac{2 \xi}{\sqrt{2 \xi}-1} e^{\xi T} \int_{0}^{\infty} \frac{(x+u)(y+u)}{A^{2}} K_{2}(\sqrt{\xi} A) d u d \xi \\
\sim-e^{-(x+y+T) / 2 \frac{2}{\pi}} \int_{0}^{\infty} \frac{(x+u)(y+u)}{A^{2}} \int_{0}^{\infty} A^{2} w^{-3} e^{-w / 2} \frac{1}{2 \pi i} \int_{\mathrm{Br}^{\prime}} \xi^{2}
\end{gathered}
$$




$$
\begin{aligned}
& \times \exp \left[\xi\left(T-\frac{A^{2}}{2 w}\right)\right] d \xi d w d u \\
& =-e^{-(x+y+T) / 2 \frac{2}{\pi}} \int_{0}^{\infty}(x+u)(y+u) \int_{0}^{\infty} w^{-3} e^{-w / 2} \delta^{\prime \prime}\left(T-\frac{A^{2}}{2 w}\right) d w d u \\
& =-e^{-(x+y+T) / 2} \frac{2}{\pi} \int_{0}^{\infty}(x+u)(y+u) \int_{0}^{\infty} v \exp \left(-\frac{1}{2 v}\right) \delta^{\prime \prime}\left(T-\frac{A^{2}}{2} v\right) d v d u \\
& =-\frac{2}{\pi} e^{-(x+y+T) / 2} \int_{0}^{\infty} \frac{1}{4 T^{3}}(x+u)(y+u) \exp \left[-\frac{(x+u)^{2}+(y+u)^{2}}{4 T}\right] d u \\
& =-\frac{1}{2 \pi T^{3}} e^{-(x+y+T) / 2} J .
\end{aligned}
$$

This gives the leading term in Theorem 2, part $(i)$. From the above, it is easy to show that $J=O\left(T^{3 / 2}\right)$ for $x, y=O(\sqrt{T})$, and the integral cannot be further simplified in this limit. We also have

$$
\begin{aligned}
J=J(x, y, T) & \sim x y \frac{2 T}{x+y} \exp \left[-\frac{x^{2}+y^{2}}{4 T}\right], \frac{x^{2}+y^{2}}{T} \rightarrow \infty \\
J & \sim T^{3 / 2} \sqrt{\frac{\pi}{2}}, \frac{x^{2}+y^{2}}{T} \rightarrow 0 .
\end{aligned}
$$

Comparing this with the expansions for $x, y=O(T)$ and $x, y=O(1)$, we see that the expansion on the intermediate scale matches properly to the other two. To compute the correction term in part $(i)$ that arises from $J_{1}$ we simply refine the small $\xi$ approximation of the integrand to $2 \xi /(\sqrt{2 \xi}-1)=-2 \xi-2 \sqrt{2 \xi^{3 / 2}}+O\left(\xi^{2}\right)$. Then a calculation completely analogous to $(5.12)$ yields the term proportional to $J_{1}$ in Theorem $2(i)$. We also obtain the asymptotic result for $x, y=O(\sqrt{T})$ by using representation (3.3) for $P_{I I}$. This completes the proof of Theorem 2 .

Next we consider $\rho>1$ and set $a=-1$ and $x_{0}=y_{0}=0$. The proof of Theorem 3 is similar to that of Theorem 2. The main differences are that the factor $e^{-(x+y) / 2}$ in $(5.3)$ must be replaced by $e^{(x+y) / 2}$, and $\sqrt{2 \xi}-1$ in the integrand's denominator must be replaced by $\sqrt{2 \xi}+1$. The saddle point is as in (5.4) and (5.5), but now the pole at $\xi=1 / 2$ is absent. Thus, the saddle point approximation $P \sim L e^{\Phi}$ is valid in the entire interior of the $(x, y, T)$ space. The face asymptotics are again obtained (for $y=O(1)$ ) by using (5.6) and the saddle point method to evaluate the integral over $\theta$ (or $\xi$ ). The edge asymptotics follow from (5.7)-(5.12), after the two changes noted above.

When $\rho=1$, we set $a=0$ and obtain

$$
\begin{aligned}
P= & \frac{2 \sqrt{2}}{\pi} \frac{1}{2 \pi i} \int_{\mathrm{Br}} \sqrt{\theta} e^{\theta T} \int_{0}^{\infty} \frac{(x+u)(y+u)}{(x+u)^{2}+(y+u)^{2}} \\
& \times K_{2}\left(\sqrt{\theta} \sqrt{(x+u)^{2}+(y+u)^{2}}\right) d u d \theta .
\end{aligned}
$$


Applying the representation for $K_{2}(\cdot)$ above equation (5.12), to (5.13) we can explicitly evaluate the integral over $\theta$. Then, after some elementary manipulation, we are left with a double integral, which is the same as that in Theorem $4(e)$. The other parts of Theorem 4 are easily established using either the last integral, or (5.13) and the saddle point method.

Finally, we briefly discuss the proof of Theorems $2^{*}-4^{*}$. For fixed $\left(x_{0}, y_{0}\right)$, the initial conditions in (3.5) appear only in the factor $\exp \left[\left(x_{0}+y_{0}\right)\left(\frac{a}{2}-\sqrt{\frac{\theta}{2}+\frac{a^{2}}{4}}\right)\right]$, and this does not affect the location(s) of the saddle points. This factor, when evaluated at $\theta=\xi_{0}-1 / 2$, multiplies the function $K$ in Theorem $2^{*}(a)$. The other terms arise from the expansion of $P_{I}$ in (3.2). The calculation of these terms is routine, as it only involves the asymptotic expansion of the integrals in (3.2). These may be expressed in terms of standard error functions whose asymptotic properties are well established. The functions $J_{2}$ and $J_{3}$ in Theorem $2^{*}(i)$ arise from $P_{I}$. In the limit $T \rightarrow$ $\infty$, with $x, y=O(\sqrt{T})$, these integrals cannot be explicitly evaluated.

Thus, we have given very detailed asymptotic results for large space/time scales, but fixed initial conditions $\left(x_{0}, y_{0}\right)$. If $x_{0}$ and/or $y_{0}$ are also large, the asymptotics become invalid, as the locations of the various saddle points now also depends on initial conditions.

Further interesting insights into the behavior of the density $P\left(x, y, T ; x_{0}, y_{0}\right)$ can be seen by considering large initial conditions, and we hope to accomplish it in subsequent work. For example, when $x_{0}, y_{0}$ are both large, and $(x, y)$ is sufficiently far from the boundaries, we can approximate $P_{I}$ by the first of the four terms inside $\{\ldots\}$ in (3.2). This corresponds to a free space, two-dimensional diffusion process and for certain ranges of $(x, y, T)$ (e.g. short times) we have $P_{I} \gg P_{I I}$ and thus $P \sim P_{I}$. We can view the other three terms inside $\{\ldots\}$ as interactions with one of the two boundaries, and $P_{I I}$ corresponds to the process interacting with both boundaries simultaneously. An asymptotic analysis for $x_{0}$ and $y_{0}$ large would better quantify the time and magnitude of the boundary interactions, as was the case with the one-dimensional models studied in $[10,18]$ and [23].

\section{Appendix A}

We obtain the density for the sum process $X(T)+Y(T)$ by evaluating the Laplace transform of $\int_{0}^{z} P(w, z-w, T) d w$. We first evaluate the contribution to this integral from $P_{I I}$, using representation (3.6). Setting $s=\theta+a^{2} / 2$ we have

$$
\begin{gathered}
\int_{-\infty}^{\infty} \frac{i \sinh (2 \eta)}{\cosh \eta+i \sinh \eta} \int_{0}^{z} \exp [-\sqrt{s}((z-w) \cosh \eta+i w \sinh \eta)] d w d \eta \\
=\int_{-\infty}^{\infty} \frac{i}{\sqrt{s}} \frac{\sinh (2 \eta)}{\cosh ^{2} \eta+\sinh ^{2} \eta}\left[e^{-i \sqrt{s} z \sinh \eta}-e^{-\sqrt{s} z \cosh \eta}\right] d \eta
\end{gathered}
$$




$$
=\frac{i}{\sqrt{s}} \int_{-\infty}^{\infty} \frac{\sinh (2 \eta)}{\cosh ^{2} \eta+\sinh ^{2} \eta} e^{-i \sqrt{s} z \sinh \eta} d \eta
$$

The last integral is easily evaluated by shifting the contour down, with $\eta=v-i \pi / 2$. The integral over the new contour vanishes, since the integrand is an odd function of $v$. Inside the strip $-\pi / 2<\operatorname{Im}(\eta)<0$, there is a pole at $\eta=-i \pi / 4$. Hence, (A.1) is equal to

$$
-2 \pi i[\text { residue at }-i \pi / 4]=\frac{\pi}{\sqrt{s}} \exp \left[-\sqrt{\frac{s}{2}} z\right]
$$

Using (A.1), (A.2), and (3.6) we obtain

$$
\int_{0}^{z} \widehat{P}_{I I}(w, z-w, T) d w=\frac{\sqrt{2 s}+a}{2 \theta} \exp \left[\left(\frac{a}{2}-\sqrt{\frac{s}{2}}\right) z_{0}-\left(\frac{a}{2}+\sqrt{\frac{s}{2}}\right) z\right]
$$

where $z_{0}=x_{0}+y_{0}$. where $z_{0}=x_{0}+y_{0}$.
Next we consider the contribution to $\int_{0}^{z} P(w, z-w, T) d w$ that comes from $P_{I}$. In
view of $(3.2)$, we write

$$
\int_{0}^{z} P_{I}(w, z-w, T) d w=\frac{e^{-a\left(z-z_{0}\right) / 2} e^{-a^{2} T / 2}}{4 \pi T}\left[\mathscr{P}_{1}+\mathscr{P}_{2}+\mathscr{P}_{3}+\mathscr{P}_{4}\right]
$$

where the $\mathscr{P}_{j}$ 's correspond to the respective contributions from the four terms inside $\{\ldots\}$ in (3.2). Thus we have

and

$$
\begin{gathered}
\mathscr{P}_{1}=\int_{-z / 2}^{z / 2} \exp \left\{-\frac{1}{4 T}\left[\left(\frac{z}{2}+w-x_{0}\right)^{2}+\left(\frac{z}{2}-w-y_{0}\right)^{2}\right]\right\} d w \\
\mathscr{P}_{2}=-\int_{-z / 2}^{z / 2} \exp \left\{-\frac{1}{4 T}\left[\left(\frac{z}{2}+w+x_{0}\right)^{2}+\left(\frac{z}{2}-w+y_{0}\right)^{2}\right]\right\} d w
\end{gathered}
$$

$$
\begin{gathered}
\int_{0}^{\mathscr{P}_{3}} \frac{x_{0}+y_{0}+2 w-z}{2 T} \int_{z / 2}^{\infty} \exp \left\{-\frac{1}{4 T}\left[\left(v+x_{0}+w-\frac{z}{2}\right)^{2}+\left(v-y_{0}+\frac{z}{2}-w\right)^{2}\right]\right\} d v d w \\
=\int_{z / 2}^{\infty} \int_{0}^{z}-\frac{\partial}{\partial w} \exp \left\{-\frac{1}{4 T}\left[\left(v+x_{0}+w-\frac{z}{2}\right)^{2}+\left(v-y_{0}+\frac{z}{2}-w\right)^{2}\right]\right\} d w d v \\
=\int_{z / 2}^{\infty} \exp \left\{-\frac{1}{4 T}\left[\left(v+x_{0}-\frac{z}{2}\right)^{2}+\left(y_{0}-v-\frac{z}{2}\right)^{2}\right]\right\} d v
\end{gathered}
$$




$$
-\int_{z / 2}^{\infty} \exp \left\{-\frac{1}{4 T}\left[\left(v+x_{0}+\frac{z}{2}\right)^{2}+\left(y_{0}-v+\frac{z}{2}\right)^{2}\right]\right\} d v
$$

Setting $v \rightarrow-v$ in the first part of $\mathscr{P}_{3}$ and adding the result to (A.5) yields

$$
\begin{gathered}
\mathscr{P}_{1}+\mathscr{P}_{2}+\mathscr{P}_{3}=\int_{-\infty}^{z / 2} \exp \left\{-\frac{1}{4 T}\left[\left(\frac{z}{2}+w-x_{0}\right)^{2}+\left(\frac{z}{2}-w-y_{0}\right)^{2}\right]\right\} d w \\
-\int_{-z / 2}^{\infty} \exp \left\{-\frac{1}{4 T}\left[\left(\frac{z}{2}+w+x_{0}\right)^{2}+\left(\frac{z}{2}-w+y_{0}\right)^{2}\right]\right\} d w .
\end{gathered}
$$

A calculation analogous to that for $\mathscr{P}_{3}$ leads to

$$
\begin{gathered}
\mathscr{\rho}_{4}=\int_{z / 2}^{\infty} \exp \left\{-\frac{1}{4 T}\left[\left(v-x_{0}+\frac{z}{2}\right)^{2}+\left(\frac{z}{2}-v-y_{0}\right)^{2}\right]\right\} d v \\
-\int_{z / 2}^{\infty} \exp \left\{-\frac{1}{4 T}\left[\left(v-x_{0}-\frac{z}{2}\right)^{2}+\left(v+y_{0}+\frac{z}{2}\right)^{2}\right]\right\} d v
\end{gathered}
$$

We set $v \rightarrow-v$ in the second part of $\Phi_{4}$ and then add (A.6) to (A.7), which gives

$$
\begin{aligned}
\mathscr{P}_{1}+\mathscr{P}_{2}+\mathscr{P}_{3}+\mathscr{P}_{4} & =\int_{-\infty}^{\infty} \exp \left\{-\frac{1}{4 T}\left[\left(w+\frac{z}{2}-x_{0}\right)^{2}+\left(w+y_{0}-\frac{z}{2}\right)^{2}\right]\right\} d w \\
& -\int_{-\infty}^{\infty} \exp \left\{-\frac{1}{4 T}\left[\left(w+\frac{z}{2}+x_{0}\right)^{2}+\left(w-y_{0}-\frac{z}{2}\right)^{2}\right]\right\} d w \\
& =\sqrt{2 \pi T}\left[\exp \left(\frac{z z_{0}}{4 T}\right)-\exp \left(-\frac{z z_{0}}{4 T}\right)\right] \exp \left[-\frac{z^{2}+z_{0}^{2}}{8 T}\right] .
\end{aligned}
$$

Using (A.8) in (A.4) and taking the Laplace transform over the time variable we obtain

$$
\int_{0}^{z} \widehat{P}_{I}(w, z-w, \theta) d w=\frac{e^{-a\left(z-z_{0}\right) / 2}}{2 \sqrt{2 s}}\left[e^{-\left|z-z_{0}\right| \sqrt{s / 2}}-e^{-\left(z+z_{0}\right) \sqrt{s / 2}}\right] .
$$

Here we have used $\mathcal{L}\left\{(T \pi)^{-1 / 2} \exp \left[-\frac{A^{2}}{8 T}\right]\right\}=\theta^{-1 / 2} \exp (-|A| \sqrt{\theta / 2}) . \quad$ By adding (A.9) to (A.3) and noting that $s=\theta+a^{2} / 2$, we obtain precisely expression (3.8). 


\section{Acknowledgement}

I would like to thank the referee(s) for their very useful suggestions.

\section{References}

[1] Avram, F., Large deviations approximation for the stationary distribution of two dimensional regulated Brownian motion, preprint.

[2] Blanc, J.P.C., The relaxation time of two queueing systems in series, Comm. Statist. Stoch. Models 1 (1985), 1-16.

[3] Bleistein, N. and Handelsman, R.A., Asymptotic Expansions of Integrals, Holt, Rinehart and Winston, New York 1975.

[4] Coffman, Jr., E.G., Muntz, R.R. and Trotter, H., Waiting time distributions for processor-sharing systems, J. Assoc. Comput. Mach. 17 (1970), 123-130.

[5] Cohen, J.W., The Single Server Queue, North-Holland, Amsterdam 1982.

[6] Fayolle, G. and Iasnogorodski, R., Two coupled processors: The reduction to a Riemann-Hilbert problem, Z. Wahrsch. 47 (1979), 325-351.

[7] Fendick, K.W. and Rodrigues, M.A., A heavy-traffic comparison of shared and segregated buffer schemes for queues with head-to-the-line processor-sharing discipline, Queueing Systems 9 (1991), 163-190.

[8] Foschini, G.J., Equilibria for diffusion models for pairs of communicating computers-symmetric case, IEEE Trans. Informat. Theory 28 (1982), 273-284.

[9] Knessl, C., On the diffusion approximation to two parallel queues with processor-sharing, IEEE Trans. Automat. Control 36 (1991), 1356-1367.

[10] Knessl, C., On the transient behavior of the Erlang loss model, Comm. Statist. Stoch. Models 6 (1990), 749-776.

[11] Konheim, A.G., Meilijson, I. and Melkman, A., Processor-sharing of two parallel lines, J. Appl. Probab. 18 (1981), 952-956.

[12] Morrison, J.A., Diffusion approximation for head-of-the-line processor sharing for two parallel queues, SIAM J. Appl. Math. 53 (1993), 471-490.

[13] Morrison, J.A., Head of the line processor sharing for many symmetric queues with finite capacity, Queueing Systems 14 (1993), 215-237.

[14] Newell, G.F., Approximate Behavior of Tandem Queues, Springer-Verlag, New York 1979.

[15] Ott, T.J., The sojourn-time distribution in the $M / G / 1$ queue with processor sharing, J. Appl. Probab. 21 (1984), 360-378.

[16] Ramaswami, V., The sojourn time in the $G I / M / 1$ queue with processor sharing, J. Appl. Probab. 21 (1984), 437-442.

[17] Sengupta, B., An approximation for the sojourn-time distribution for the GI/G/1 processor-sharing queue, Comm. Statist. Stoch. Models 8 (1992), 35-57.

[18] Tan, X. and Knessl, C., Integral representations and asymptotics for infiniteand finite-capacity queues described by the unfinished work, I and II, SIAM J. Appl. Math. 57 (1997), 791-823 and 824-870.

[19] Tan, X. and Knessl, C., A fork-join queueing model: Diffusion approximation, integral representations and asymptotics, Queueing Systems 22 (1996), 287-322.

[20] Yashkov, S.F., A derivation of response time distribution for an $M / G / 1$ processor-sharing queue, Prob. Control Inform. Theory 12 (1983), 133-148.

[21] Yashkov, S.F., The non-stationary distribution of number of calls in the 
$M / G / 1$ processor-sharing queue, Syst. Analysis and Simul. II (1988), 158-162, Math. Res. 47 (1988), Akademie-Verlag, Berlin.

[22] Yashkov, S.F., Processor-sharing queues: Some progress in analysis, Queueing Syst. Theory Appl. 2 (1987), 1-17.

[23] Xie, S. and Knessl, C., On the transient behavior of the Erlang loss model: Heavy usage asymptotics, SIAM J. Appl. Math. 53 (1993), 555-599. 


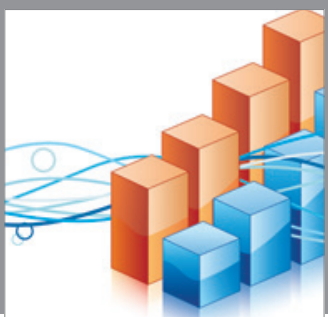

Advances in

Operations Research

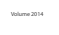

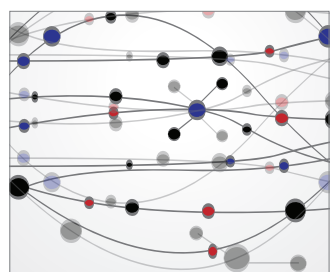

\section{The Scientific} World Journal
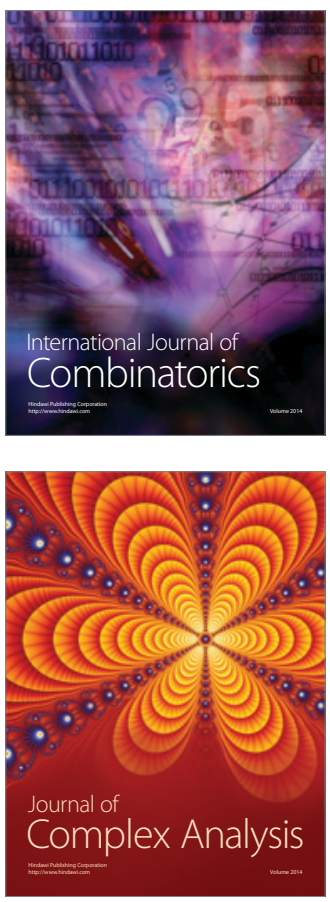

International Journal of

Mathematics and

Mathematical

Sciences
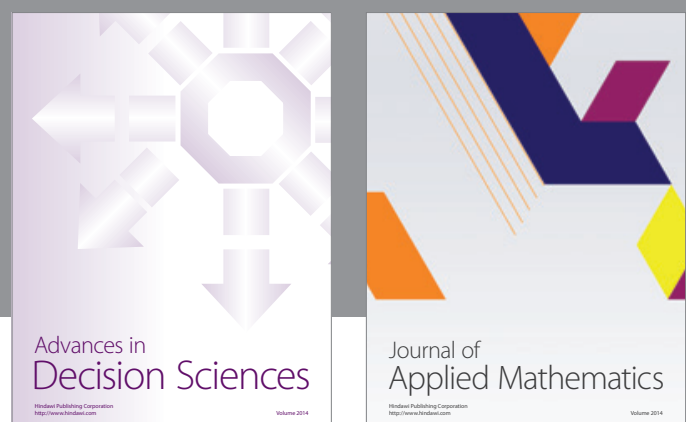

Journal of

Applied Mathematics
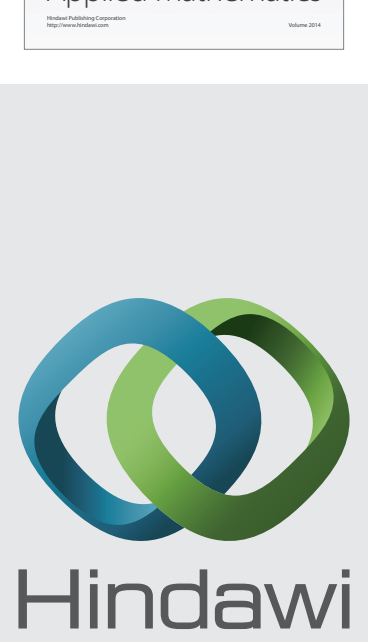

Submit your manuscripts at http://www.hindawi.com
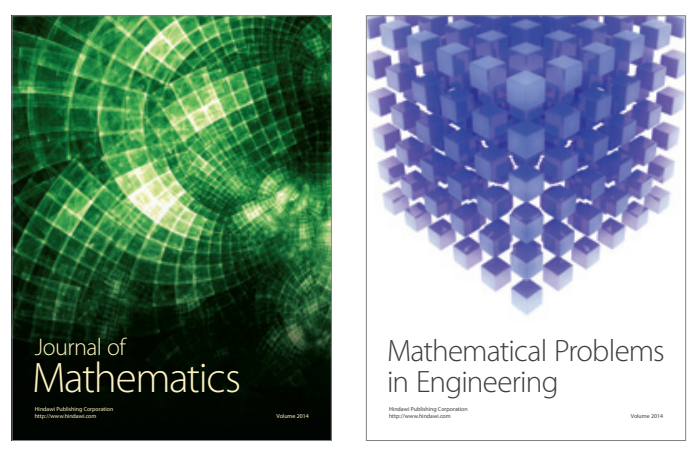

Mathematical Problems in Engineering
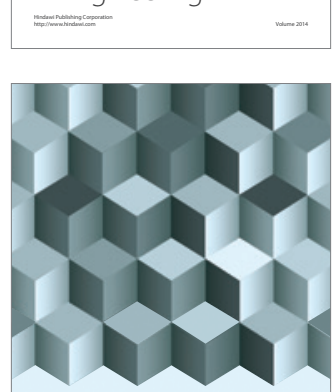

Journal of

Function Spaces
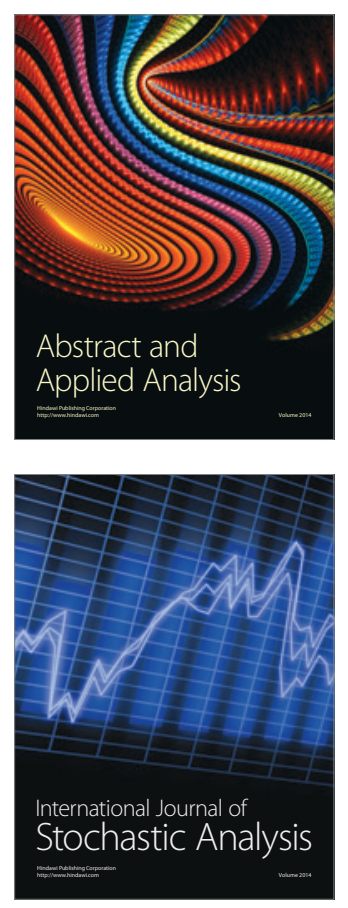

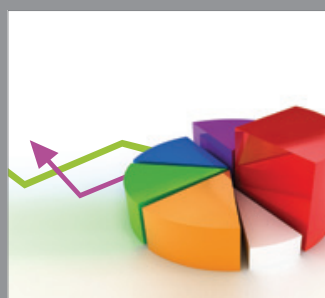

ournal of

Probability and Statistics

Promensencen
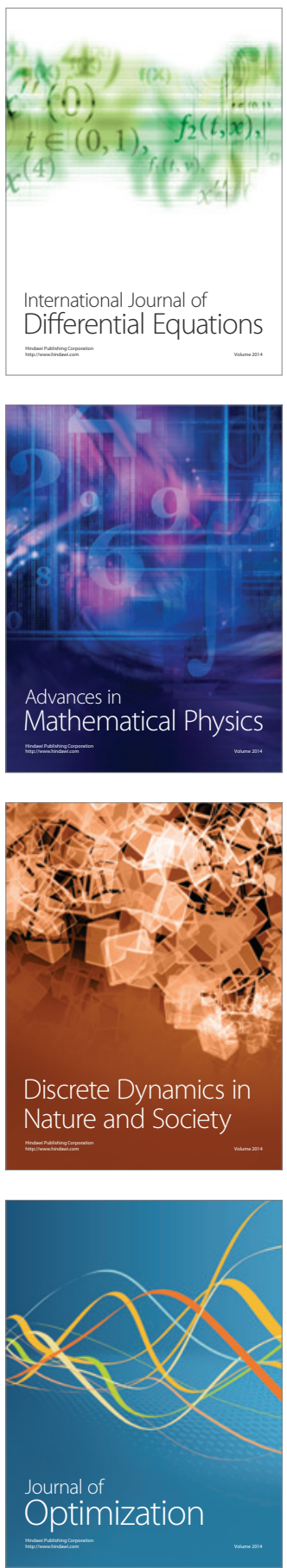